\title{
The Girsanov Linearization Method for Stochastically Driven Nonlinear Oscillators
}

For most practical purposes, the focus is often on obtaining statistical moments of the response of stochastically driven oscillators than on the determination of pathwise response histories. In the absence of analytical solutions of most nonlinear and higherdimensional systems, Monte Carlo simulations with the aid of direct numerical integration remain the only viable route to estimate the statistical moments. Unfortunately, unlike the case of deterministic oscillators, available numerical integration schemes for stochastically driven oscillators have significantly poorer numerical accuracy. These schemes are generally derived through stochastic Taylor expansions and the limited accuracy results from difficulties in evaluating the multiple stochastic integrals. As a numerically superior and semi-analytic alternative, a weak linearization technique based on Girsanov transformation of probability measures is proposed for nonlinear oscillators driven by additive white-noise processes. The nonlinear part of the drift vector is appropriately decomposed and replaced, resulting in an exactly solvable linear system. The error in replacing the nonlinear terms is then corrected through the Radon-Nikodym derivative following a Girsanov transformation of probability measures. Since the RadonNikodym derivative is expressible in terms of a stochastic exponential of the linearized solution and computable with high accuracy, one can potentially achieve a remarkably high numerical accuracy. Although the Girsanov linearization method is applicable to a large class of oscillators, including those with nondifferentiable vector fields, the method is presently illustrated through applications to a few single-and multi-degree-of-freedom oscillators with polynomial nonlinearity. [DOI: 10.1115/1.2712234]

Keywords: Girsanov transformation, Ito-Taylor expansion, multiple stochastic integrals, Monte Carlo simulation, Wiener processes, nonlinear oscillators

\section{Introduction}

The available approximate analytical methods for determination of transitional probability density or statistical moments of the response of stochastically driven nonlinear oscillators generally suffer from the dimensionality curse and/or restrictions on the type and number of stochastic excitations [1]. A much more versatile and popularly adopted route is a Monte Carlo simulation (MCS) aided by direct numerical integration of the associated stochastic differential equations (SDEs). Direct MCS, while often regarded as less elegant, can be used to solve problems of significantly higher complexity and dimensions. Even then, its implementation is hindered by the intensively repetitive computation over a possibly large ensemble and the accuracy of the direct integration scheme. Indeed, as compared to numerical integration schemes for deterministic DEs, most of the available ones for SDEs, often derived via stochastic Taylor expansions, have considerably lower orders of accuracy. This happens due to difficulties in computing the multiple stochastic integrals (MSIs) [2,3]. A few such typical schemes include the Euler-Maruyama (Maruyama [4]), stochastic Heun (Gard [5]), stochastic RungeKutta [6-8], and stochastic Newmark $[9,10]$. Moreover, there are also implicit variations of these methods [10-12] that have a higher stochastic numerical stability than their explicit counterparts. Although the implicit methods work well for even stiff SDEs, solutions tend to become inexact for very low step sizes [10]. These stochastic integration schemes may also be catego-

\footnotetext{
Corresponding author.

Contributed by the Applied Mechanics Division of ASME for publication in the Journal of ApPLIED MeCHANICS. Manuscript received April 10, 2006; final manuscript received November 3, 2006. Review conducted by N. Sri Namachchivaya.
}

rized as strong and weak. Although strong schemes are designed to simulate the pathwise unique response corresponding to a particular realization of the stochastic excitation [13], weak schemes only compute statistical moments of functions of the response by replacing the MSIs with random variables having simpler distributions [3]. Accordingly, a weak algorithm is computationally faster and the preferred choice in most engineering applications, wherein it suffices to obtain approximations to certain statistical moments or distribution functions. Nevertheless, among all these integration schemes-weak or strong, implicit or explicit- the ones with the highest accuracy are the stochastic Runge-Kutta (an explicit method of weak order 2 [8]) and the stochastic Newmark (an implicit method of weak order 3 [10]).

Among the analytical or numeric-analytic techniques, linearization methods (the equivalent linearization method in particular) have found a certain measure of acceptability. Recently, Socha $[14,15]$ has provided a review of equivalent and statistical linearization in the analysis of nonlinear oscillators. Equivalent linearization methods essentially try to replace the nonlinear function by an equivalent linear one such that the error in the replacement is minimized in a sense. The approximating linear function may be obtained through several criteria, e.g., mean square approximation $[16,17]$, errors in response moments [18], energy [19], frequency [20], probability density [21], and an improved version of Gaussian equivalent linearization [22]. These methods generally use iterative procedures for the determination of the equivalent linear function and, subsequently, response statistics. Such statistical linearization methods have been applied to such applications as filtering, control [23] and computation of response statistics of hysteretic oscillators [24]. It is however known that the class of global linearization methods, although very efficient when it works, is unacceptably inaccurate for most nonlinear single- and 
multi-degree-of-freedom (SDOF and MDOF) systems. Other than globally defined equivalent linearization schemes, mention may also be made of a few other forms of stochastic linearizations. These include conditional linearization [25] and local forms of linearizations, viz. the phase space linearization [26] and the locally transversal linearization $[27,28]$. Although conditional linearization lacks universal applicability, the others are limited in numerical accuracy. Indeed, the observation on the lack of general applicability is valid for several other analytical tools, such as the method of moment closures [29], stochastic averaging [30], maximum entropy [31] etc. Moreover, the quality of approximation via all these methods in the general context of nonlinear oscillators is very often poor.

A weak and numeric-analytic method of stochastic linearization, referred to as the Girsanov linearization method (GLM), is presently proposed for nonlinear oscillators under additive stochastic excitations. The essence of the GLM is an appropriate linearized replacement of the nonlinear drift terms followed by an appeal to the Girsanov transformation of probability measures to weakly correct for the error caused due to this replacement. This correction is multiplicative in nature, satisfies a scalar SDE that is "exactly" solvable in terms of the linearized solution and is referred to as the Radon-Nikodym derivative (also called the likelihood ratio). In particular, the solution for the likelihood ratio is in the form of a stochastic exponential whose argument is in the form of integrals of the linearized solution. However, the numerical computation of the stochastic exponential is generally prone to considerable numerical instability and forms the most crucial part of the development of the GLM. The present framework developed to arrest the numerical instability requires, as a first step, that the time interval of interest be discretized into smaller subintervals and the linearization performed over each such subinterval. Two versions of the GLM then evolve depending on how the stochastic exponential is computed over each subinterval. Although version I uses a stochastic Taylor expansion for an approximate evaluation of the exponential, version II does it in such a way that the error is introduced only due to the finiteness of the ensemble size. Other than the semi-analyticity and unparalleled numerical accuracy, yet another useful feature of the GLM is its ability to treat oscillators even with nondifferentiable nonlinearity. Nevertheless, only a limited numerical illustration of the GLM is presently provided through its application to a few singleand two-degree-of-freedom (2-DOF) oscillators with polynomial nonlinearity.

\section{Girsanov Linearization}

As mentioned earlier, present study restricts itself to the derivation of the GLM in the context of nonlinear (mechanical) oscillators while keeping in mind that the method may be readily extended to a more general class of nonlinear SDEs. Thus consider the equations of motion of an $n$-DOF oscillator excited by white noise processes in the following form:

$$
[M] \ddot{X}+C \dot{X}+K X+\chi(X, \dot{X}, t)=\sum_{r=1}^{q} G_{r}(t) \dot{W}_{r}+F(t)
$$

where $X=\left\{x^{(1)}, x^{(2)}, \ldots, x^{(n)}\right\}^{T} \in \mathbf{R}^{n}$ is the displacement vector. Equation (1) is subject to the initial condition $X(t=0):=X_{0}$ $=\left\{x_{0}^{(1)}, x_{0}^{(2)}, \ldots, x_{0}^{(n)}\right\}^{T},[M][C],[K] \in \mathbf{R}^{n \times n}$ are constant mass, damping, and stiffness matrices, respectively, $\chi(X, \dot{X}, t)$ is any vector function (not necessarily smooth) that ensures a unique solution of Eq. (1) at least in the weak sense, $\left\{G_{r}(t): \mathbf{R} \rightarrow \mathbf{R}^{n}\right\}$ is the set of $n$ diffusion vectors (additive), $\left\{W_{r}(t) \mid r \in[1, q]\right\}$ is a $q$-dimensional vector of independently evolving zero-mean Wiener processes with $W_{r}(0)=0, \mathbf{E}\left[\left|W_{r}(t)-W_{r}(s)\right|^{2}\right]=(t-s), t>s$ $\forall r \in[1, q]$ and $F(t)=\left\{F^{(j)}(t) \mid j=1, \ldots, n\right\}$ is the externally applied deterministic force vector. $\mathbf{E}$ denotes the expectation operator with respect to the underlying probability measure $P$. The description of the oscillator as in Eq. (1) being entirely formal (due to nondifferentiability of Wiener processes, which implies that $\dot{W}_{r}(t)$ exists merely as a valid measure, but not as a mathematical function), they may more appropriately be recast as a system of $2 n$ first order SDEs in the following incremental form:

$$
\begin{gathered}
d x_{1}^{(j)}=x_{2}^{(j)} d t \\
d x_{2}^{(j)}=a^{(j)}(X, \dot{X}, t) d t+\sum_{r=1}^{q} b_{r}^{(j)}(t) d W_{r}(t), \quad j=1,2, \ldots, n
\end{gathered}
$$

where

$$
\begin{gathered}
a^{(j)}(X, \dot{X}, t)=-\sum_{k=1}^{n} \hat{C}_{j k} \dot{x}^{(k)}-\sum_{k=1}^{n} \hat{K}_{j k} x^{(k)}-\hat{\chi}^{(j)}(X, \dot{X}, t)+\hat{F}^{(j)}(t) \\
{[\hat{C}]=\left[M^{-1}\right][C], \quad[\hat{K}]=\left[M^{-1}\right][K],} \\
\{\hat{F}\}=\left[M^{-1}\right]\{F\}, \quad\{\hat{\chi}\}=\left[M^{-1}\right]\{\chi\} \\
B_{r}(t)=\left\{b_{r}^{(j)}(t) \mid j=1, \ldots, n\right\}=\left[M^{-1}\right] G_{r}(t)
\end{gathered}
$$

Moreover, define $\bar{X}_{0}=\left\{x_{1,0}^{(1)}, x_{2,0}^{(1)}, x_{1,0}^{(2)}, x_{2,0}^{(2)}, \ldots, x_{1,0}^{(n)}, x_{2,0}^{(n)}\right\}^{T} \in \mathbf{R}^{2 n}$. Now we assume that the drift vector $A=\left\{a^{(j)} \mid j=1, \ldots, n\right\}$ (coefficient of $d t$ term) can be decomposed into two constituent parts as $a^{(j)}=a_{l}^{(j)}+a_{n l}^{(j)}$ (i.e., $A=A_{l}+A_{n l}$ ), where $A_{l}$ denotes the linear part of the vector field that should remain in the linearized equations following GLM and $A_{n l}$ denotes the nonlinear part. To ensure boundedness of the solution vector $\bar{X} \triangleq\left\{x_{1}^{(1)}, x_{2}^{(1)}, x_{1}^{(2)}\right.$, $\left.x_{2}^{(2)}, \ldots, x_{1}^{(n)}, x_{2}^{(n)}\right\}^{T} \in \mathbf{R}^{2 n} \quad \forall t \quad$ (such that $X_{1}:=X=\left\{x_{1}^{(j)}\right\}^{T} \in \mathbf{R}^{n}$, $X_{2}:=\dot{X}=\left\{x_{2}^{(j)}\right\}^{T} \in \mathbf{R}^{n}$ ) and uniqueness in a weak sense, it is assumed that the drift and diffusion vectors, $A=\left\{a^{(j)}\right\}$ and $B_{r}$ $=\left\{b_{r}^{(j)}\right\}$ are measurable and satisfy the following bounds:

$$
\begin{gathered}
\|A(\bar{X}, t)\|+\left\|\sum_{r=1}^{q} B_{r}(t)\right\| \leqslant Q_{1}(1+\|\bar{X}\|) \\
\|A(\bar{X}, t)-A(\bar{Y}, t)\| \leqslant Q_{2}\|\bar{X}-\bar{Y}\|
\end{gathered}
$$

where $\bar{Y} \in \mathbf{R}^{2 n}, Q_{1}, Q_{2} \in R^{+}$and $\|$.$\| denotes the Euclidean norm$ (i.e., the $L_{2}(P)$ norm in the associated probability space $\left.\left[\Omega, F_{t}, P\right]\right)$. Let the initial conditions be mean square bounded, i.e., $\mathbf{E}\left\|\bar{X}\left(t_{0}\right)\right\|^{2}<\infty$ (without a loss of generality, the initial condition vector is presently treated as deterministic). The time interval $[0, T]$ of interest is so ordered (discretized) that $0=t_{0}<t_{1} \cdots<t_{i}$ $<\cdots<t_{L}=T, h_{i}=t_{i}-t_{i-1}$, and $T_{i}=\left(t_{i-1}, t_{i}\right]$, where $i \in Z^{+}$. For further simplification of the rest of the presentation without a loss of focus on the main issues, we assume a uniform time step $h_{i}$ $=h \forall i$ unless otherwise stated.

We are in interested in computing the weak response, i.e., expectations of the form

$$
v=\mathbf{E}[f(\bar{X}, t)]
$$

where $f(\cdot)$ denotes some real-valued function. When we utilize Monte Carlo simulation to evaluate the expectation, $v$ is replaced by its sample-mean formula [32]

$$
v \approx \hat{v}=\frac{1}{N} \sum_{m=1}^{N}\left[f\left(\bar{X}^{(m)}, t\right)\right] \text { for large } N
$$

where $\bar{X}^{(m)}(t)$ denotes the $m$ th sample path of $\bar{X}(t)$ and $N$ is the number of sample paths (i.e., the ensemble size). Since it suffices to evaluate $\hat{v}$ using weak solutions of Eq. (1), we intend to use a measure transformation based on Girsanov's theorem [2] to locally linearize Eq. (1) and thus (hopefully) obtain highly accurate 
estimates of the required expectation based on the known analytical solutions of the linear SDEs. By "local linearization," we mean that the governing SDEs would be replaced by a system of linearized ones so that the $i$ th such linearized SDE could serve as an accurate replacement for the nonlinear SDE over $T_{i}$.

Briefly, the Girsanov theorem implies that if the drift coefficient of an Ito process (with a nondegenerate diffusion coefficient) is altered to an extent (such that the original and altered drift coefficients satisfy the Novikov condition, as defined below), then the law of the process would not be altered drastically. Indeed, the law of the drift-modified process will remain absolutely continuous with respect to that of the original process and we can explicitly compute the Radon-Nikodym derivative (see the Radon-Nikodym theorem below). Before going into the formulation of GLM we state a few definitions and theorems that would be useful during the formulation.

DEFINITION ( ABSOlute CONTINUITY OF MEASURES). Let $\left[\Omega, \mathrm{F},\{\mathrm{F}\}_{t=0} P\right]$ is a filtered probability space (i.e., $[\Omega, \mathrm{F}, P]$ is a probability space and $\{\mathrm{F}\}_{t \geqslant 0}$ is a filtration on $[\Omega, P]$; presently, it is the filtration generated by the Brownian motion processes). If we fix $T>0$ and let $Q$ be another probability measure on $\{\mathrm{F}\}_{T}$, then we say that $Q$ is absolutely continuously with respect to $\left.P\right|_{F_{T}}$ (the restriction of $P$ to $\mathrm{F}_{T}$ ) and we write $Q \ll P$ if $P(H)$ $=0 \Rightarrow Q(H)=0$ for all $H \in F_{T}$.

RADON-NIKODYM THEOREM. Let the probability measure $Q$ be absolutely continuous with respect to probability measure $P$, then there exists a random variable $\Lambda(\omega) \geqslant 0$; such that $\Lambda(\omega)$ is $\mathrm{F}_{T}$ measurable, $\mathbf{E}_{P}[\Lambda(\omega)]=1$ and $Q(H)=\int_{H} \Lambda(\omega) d P$ for any measurable set $H$. In that case we write $d Q / d P=\Lambda(\omega)$ where, $\Lambda(\omega)$ is the Radon-Nikodym derivative or the likelihood ratio.

DEFINITION (A MARTINGALE). A stochastic process $\left\{\mathrm{M}_{\mathrm{t}}\right\}_{t \geqslant 0}$ on $[\Omega, \mathrm{F}, P]$ of dimension $n$ is called a martingale with respect to filtration $\{\mathrm{F}\}_{t \geqslant 0}$ (and with respect to $P$ ) if

i. $M_{t}$ is $\{\mathrm{F}\}_{t \geqslant 0}$ measurable for all $t$

ii. $E\left[\left|M_{t}\right|\right]<\infty$ for all $t$

iii. $E\left[M_{s} \mid M_{t}\right]=M_{t}$ for all $s \geqslant t$

The Martingale property. Suppose $Q \ll P$ with $d Q / d P=\Lambda(\omega)$ on $F_{T}$ (filtration generated by the Brownian motion processes). Then $Q \ll P$ for all $t \in[0, T]$ and if we define $M_{t}=d(Q) / d(P)$, then $M_{t}$ is a martingale with respect to $F_{t}$ and $P$.

Although a linearization of nonlinear oscillators using the Girsanov theorem could be nonuniquely performed, an obvious and rather tempting choice is to arrive at the linearized equations by simply removing $A_{n l}$ from Eq. (2). If this route is adopted, then the transformed (linearized) SDEs over $T_{i}$ take the form

$$
\begin{aligned}
d \widetilde{x}_{1}^{(j), i}= & \widetilde{x}_{2}^{(j), i} d t \\
d \widetilde{x}_{2}^{(j), i}= & a_{l}^{(j)}\left(\tilde{X}^{i}, \dot{\widetilde{X}}^{i}, t\right) d t \\
& +\sum_{r=1}^{q} b_{r}^{(j)}(t) \underbrace{\left\{\left[b_{r}^{(j)}(t)\right]^{-1} a_{n l}^{(j)}\left(\tilde{X}^{i}, \dot{\tilde{X}}^{i}, t\right) d t+d W_{r}(t)\right.}_{d \hat{W}_{r}^{i}(t)}\} \\
& j=1,2, \ldots, n
\end{aligned}
$$

where $\left\{\hat{W}_{r}^{i}(t) \mid r \in[1, q]\right\}$ is another vector of $q$-dimensional Brownian motion process (that absorbs the nonlinear part, $A_{n l}$, of the drift field) by the Levy characterization of Brownian motion [13] under a new probability measure $Q$. Note that $\left\{\hat{W}_{r}^{i}(t)\right\}$ as well as the linearized displacement and velocity processes, $\tilde{X}_{1}^{i}(t)$ $:=\left\{\widetilde{x}_{1}^{(j), i} \mid j \in[1, n]\right\}$ and $\tilde{X}_{2}^{i}(t):=\left\{\widetilde{x}_{2}^{(j), i} \mid j \in[1, n]\right\}$, are restricted to the interval $T_{i}$ as indicated by the superscript $i$. Equation (7) is subject to initial conditions $\tilde{\bar{X}}^{i}\left(t_{i-1}\right)=\tilde{\bar{X}}^{i-1}\left(t_{i-1}\right), i>1$ so that
$\widetilde{\bar{X}}^{1}\left(t_{0}\right)=\bar{X}\left(t_{0}\right) \quad$ for $\quad i=1$. Moreover, we define $\tilde{\bar{X}}^{i}$ $=\left\{\widetilde{x}_{1}^{(1), i}, \widetilde{x}_{2}^{(1), i}, \ldots, \widetilde{x}_{1}^{(n), i}, \widetilde{x}_{2}^{(n), i}\right\}^{T}$. Now, to compensate for the removal of nonlinear drift terms, we need to augment the linearized SDEs with the one-dimensional correction process $y^{i}(t)$ (i.e., the $i$ th Radon-Nikodym derivative) that satisfies the following scalar SDE:

$$
d y^{i}=\sum_{j=1}^{n} \sum_{r=1}^{q}\left[b_{r}^{(j)}(t)\right]^{-1} a_{n l}^{(j)}\left(\tilde{X}^{i}, \dot{\widetilde{X}}^{i}, t\right) y^{i} d \hat{W}_{r}^{i}(t)
$$

subject to the known initial condition $y^{i}\left(t_{i-1}\right)=y^{i-1}\left(t_{i-1}\right)$ so that $y^{1}\left(t_{0}\right)=1.0$ for $i=1$. Toward a simpler presentation, we henceforth remove the superscript $i$ provided that the interval $T_{i}$ is unambiguously clear from the context. Note that an exact analytical solution to the linearized SDEs corresponding to Eq. (7) is readily available [2] irrespective of the dimension $2 n$, and this solution is independent of $y(t)$. Indeed, a very attractive feature of the GLM is that it can exploit the available information on the solutions of linear SDEs - an area that is well researched and developed. If one writes Eqs. (7) and (8) in terms of the drift-modified Brownian motion processes $\hat{W}_{r}(t)$ for each $r$, then it is clear that the resulting SDEs are linear in $\tilde{\bar{X}}$. Equation (7) may then be written in the new probability law $(\widetilde{Q})$ as

$$
d \tilde{\bar{X}}=\tilde{A} \tilde{\bar{X}} d t+\bar{F}(t) d t+\sum_{r=1}^{q}\left[B_{r}(t)\right] d \hat{W}_{r}(t)
$$

where

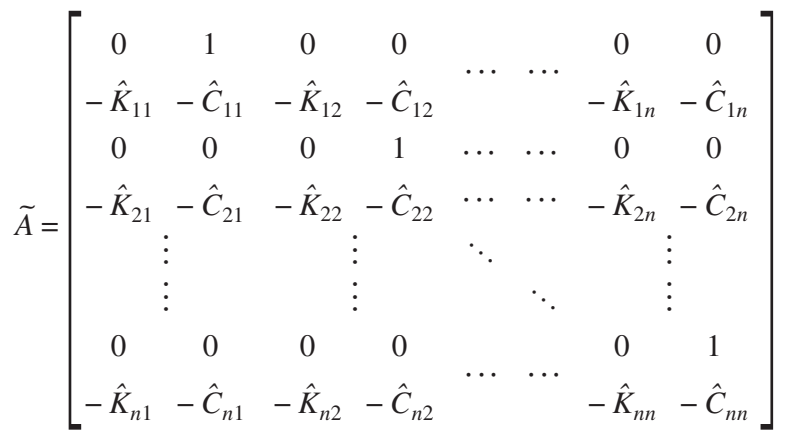

is the system matrix of the GLM-based linearized Eq. (7). Note that $\hat{K}_{j k}$ and $\hat{C}_{j k}$ are the elements of stiffness and damping matrices and $\bar{F}(t) \in \mathbf{R}^{2 n}$ is obtainable from the normalized forcing vector $\hat{F}(t) \in \mathbf{R}^{n}$ (Eq. (3)) by augmenting the latter with zeros at locations of the displacement components. The solution of Eq. (9) with the initial condition vector $\tilde{\bar{X}}_{i-1}^{i}:=\tilde{\bar{X}}^{i-1}\left(t_{i-1}\right)$ is of the form

$$
\begin{aligned}
\tilde{\bar{X}}(t)= & \exp \left(\tilde{A} \int_{t_{i-1}}^{t} d s\right) \tilde{\bar{X}}_{i-1}+\exp \left(\tilde{A} \int_{t_{i-1}}^{t} d s\right) \\
& \times \int t_{t_{i-1}}^{t}\left[\exp \left(-\tilde{A} \int_{t_{i-1}}^{s} d s_{1}\right) \bar{F}^{(j)}(t)\right] d s+\exp \left(\tilde{A} \int_{t_{i-1}}^{t} d s\right) \\
& \times \int t_{t_{i-1}}^{t}\left[\exp \left(-\tilde{A} \int_{t_{i-1}}^{s} d s_{1}\right) \sum_{j=1}^{n} \sum_{r=1}^{q}\left[b_{r}^{(j)}(t)\right] d \hat{W}_{r}(s)\right.
\end{aligned}
$$

The Radon-Nikodym derivative $y(t)$ is a strictly positive random process (an exponential martingale) computable as 


$$
\begin{aligned}
y(t)= & y_{i-1} \exp \left\{\int_{t_{i-1}}^{t} \sum_{j=1}^{n} \sum_{r=1}^{q}\left[b_{r}^{(j)}(t)\right]^{-1} a_{n l}^{(j)}\left(\widetilde{X}_{1}, \widetilde{X}_{2}, t\right) d W_{r}(s)\right. \\
& \left.-\frac{1}{2} \int_{t_{i-1}}^{t}\left[b_{r}^{(j)}(t)\right]^{-2}\left[a_{n l}^{(j)}\left(\widetilde{X}_{1}, \widetilde{X}_{2}, t\right)\right]^{2} d s\right\}
\end{aligned}
$$

Computations of required expectations may now be accomplished in terms of the GLM-based linearized solution as: $\mathbf{E}\left[f\left(\bar{X}_{i}, t_{i}\right)\right]$ $=\mathbf{E}\left[y_{i} f\left(\tilde{\bar{X}}_{i}, t_{i}\right)\right]$, subject to a localized form of Novikov's restrictions on $A_{n l}$ (i.e., $\left.\mathbf{E}\left[\exp \left(\frac{1}{2} \int_{t_{i-1}}^{t} A_{n l}^{2} d s\right)\right]<\infty\right)$ for $t \in T_{i}$.

Unfortunately, even if one chooses a very small step size, it is generally not possible to develop the above idea into a numerically stable algorithm. One may expect numerical instabilities in the form of the computed moment histories underflowing or overflowing along with widely varying oscillations. Reasons behind this may be traced to the fact that, for the Girsanov theorem to be valid, expectations of the strictly positive martingales $\left\{y^{i}(t)\right\}$ should be exactly equal to $1.0 \forall i \in \mathbf{Z}^{+}$[3]. For instance, if the drift nonlinearity is such that it affects the solution significantly away from that corresponding to the linear SDE (i.e., the SDE without $\left.A_{n l}\right)$, then sample paths of $\left\{y^{i}(t)\right\}$ would oscillate considerably away from 1 . These fluctuations imply that the computed expectation of $y^{i}(t)$ for a given $i$ would generally be quite different from 1.0 for finite (possibly small) ensemble sizes. Moreover, these errors would also propagate very fast in time, as explained in the following. Restricting attention to the interval $T_{i}$, we note that the argument of $y^{i}(t)$ may be expanded in an Ito-Taylor's series (each term of which is a zero-mean Gaussian Ito integral) so that $y^{i}(t)$ essentially admits an expression in the form of an infinite product of log-normal (exponentiated Gaussian) random variables. However, owing to a truncation of the Ito-Taylor expansion after a certain number of terms, this product would remain finite for all practical purposes. Moreover, as we progress over successive time intervals, $y(t)$ is updated once more through exponentiation, i.e., according to the identity: $y^{i}(t)=\zeta(t) \prod_{k=1}^{i-1} y_{k}^{k}$, where $\zeta(t)$ is the stochastic exponential that appears as the coefficient of $y_{i-1}^{i-1}$ in Eq. (11). Although the construction of $y(t)$ and the linear solution through exponential functions allows the GLM to be developed into a Lie group method, evaluations of such functions require more floating point operations thereby leading to higher errors. Thus, it is nearly impossible to precisely satisfy the requirement $\mathbf{E}\left[y^{i}(t)\right]=1$ especially for sufficiently large $i$ as the errors also accumulate exponentially. In other words, given that $y(t)$ is composed purely of exponential functions, there are just two possibilities, viz. either $\lim _{i \rightarrow \infty} \mathbf{E}\left[y^{i}(t)\right]=0$ or $\lim _{i \rightarrow \infty} \mathbf{E}\left[y^{i}(t)\right]=\infty$.

Figure 1(a) shows typically zero-mean Gaussian and (the corresponding) log-normal probability density functions (PDF) and Fig. 1(b) shows two sample paths of typically cumulative products of log-normal random variables-while one of these paths overflows, the other underflows. It is evident (Fig. 1(a)), unlike the Gaussian PDF (G-PDF) that is symmetric $\sim 0$, the log-normal density is not symmetric $\sim 1.0$. Indeed, clustering of the realizations of such a log-normal variable (generated from a zero-mean Gaussian variable) is more toward the left of 1 than to the right. Thus, it is this asymmetry of the log-normal PDF that causes paths of $y^{i}(t)$ underflow more often than overflow as $i \rightarrow \infty$. The same reasoning also convinces us about the near impossibility of arriving at a GLM-based linearized drift field uniformly valid over the entire time interval of interest because this would create very large fluctuations in the paths of $y(t)$ away from 1.0. In other words, the GLM has to be applied along with a discretization of the time axis and it is thus a numeric-analytic procedure for SDEs. These are
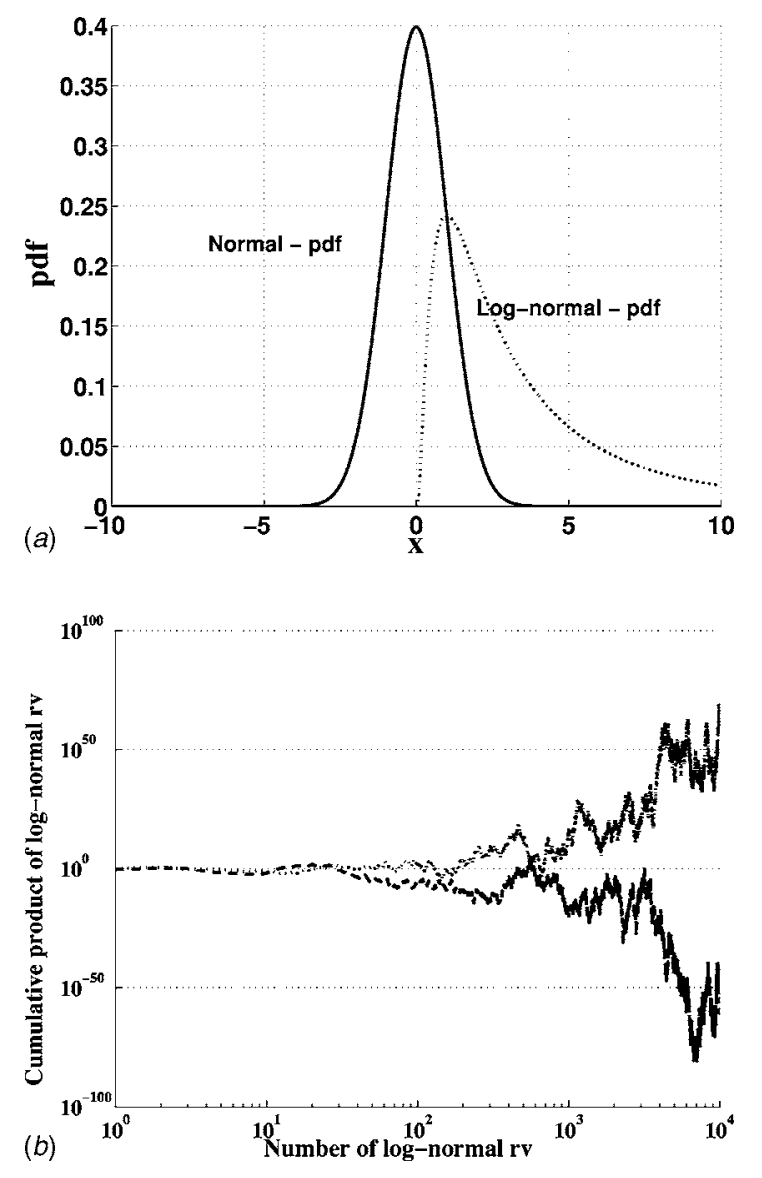

Fig. 1 (a) Typical PDFs of Gaussian and log-normal distributions and $(b)$ the cumulative product of lognormal random variables

probably the reasons why methods based on Girsanov's measure transformation have hardly been applied for stochastically driven nonlinear oscillators of engineering interest.

In order to circumvent such numerical difficulties in the GLM, it is then required to modify the linear SDEs that provide a solution "close" to that of the nonlinear SDEs, at least locally (i.e., within a given subinterval $T_{i}$ ). This would, in turn, reduce the oscillations (away from 1.0) in the sample paths of $y^{i}(t)$ to a considerable extent. This is presently achieved by locally introducing a "subtracting set" $S^{i}$, whose elements are simply various terms in the stochastic Taylor (Ito-Taylor) expansion of the nonlinear vector function $A_{n l}^{i}\left(\tilde{X}_{1}, \tilde{X}_{2}, t\right)$ (restricted to $\left.T_{i}\right)$ about $A_{n l}^{i-1}\left(t_{i-1}\right):=A_{n l, i-1}$. Moreover, these elements are hierarchically arranged in the same way as they appear in the Ito-Taylor series. Taking, for instance, the $j$ th scalar component $a_{n l}^{(j), i}\left(\widetilde{X}_{1}, \widetilde{X}_{2}, t\right)$ of $A_{n l}^{i}\left(\tilde{X}_{1}, \tilde{X}_{2}, t\right)$, its Ito-Taylor expansion based at $a_{n l, i-1}^{(j)}$ is of the form

$$
\begin{aligned}
a_{n l}^{(j), i}= & a_{n l, i-1}^{(j)}+\sum_{k=1}^{n} \sum_{r=1}^{q} b_{r}^{(k)} \frac{\partial a_{n l, i-1}^{(j)}}{\partial x_{2}^{(k)}}\left(\sqrt{t-t_{i-1}}\right) \\
& +\left[\frac{\partial}{\partial t} a_{n l, i-1}^{(j)}+\sum_{k=1}^{n}\left(x_{2}^{(k)} \frac{\partial}{\partial x_{1}^{(k)}}+a_{i-1}^{(k)} \frac{\partial}{\partial x_{2}^{(k)}}\right) a_{n l, i-1}^{(j)}\right. \\
& \left.+\frac{1}{2} \sum_{k, l=1}^{n} \sum_{r, s=1}^{q} b_{r}^{(k)} b_{s}^{(l)} \frac{\partial^{2} a_{n l, i-1}^{(j)}}{\partial x_{2}^{(k)} \partial x_{2}^{(l)}}\right]\left(t-t_{i-1}\right)+\cdots
\end{aligned}
$$

The subtracting set is of the form: $S^{i}=\left\{S_{1}^{i}, S_{2}^{i}, S_{3}^{i}, \ldots\right\}$, where, $S_{1}^{i}$ 


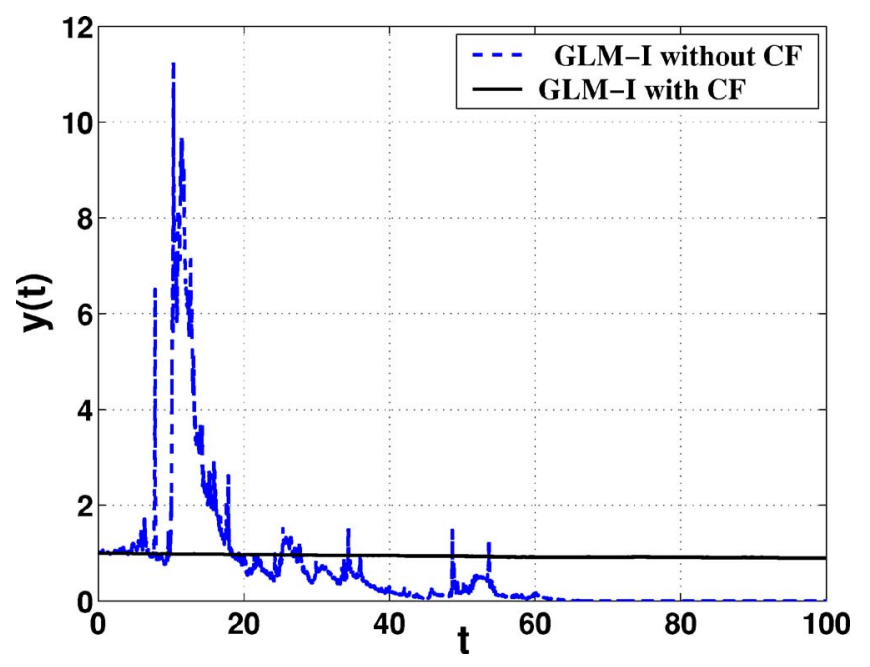

Fig. 2 A realization of the process $y(t)$ with and without the CF

$=A_{n l, i-1}, S_{2}^{i}=\sum_{k=1}^{n} \sum_{r=1}^{q} b_{r}^{(k)} \partial a_{n l, i-1}^{(j)} / \partial x_{2}^{(k)}\left(\sqrt{t-t_{i-1}}\right)$, and so on. Thus, with the incorporation of the elements of the subtracting set as hierarchically corrective forcing terms we rewrite Eq. (2) as

$$
\begin{aligned}
d \bar{X}= & \tilde{A} \bar{X} d t+\bar{F}(t) d t+\bar{\gamma}_{v} d t+\sum_{r=1}^{q} B_{r}(t)\left\{B_{r}^{-1}(t)\left[A_{n l}(X, \dot{X}, t)-\bar{\gamma}_{v}\right] d t\right. \\
& \left.+d \hat{W}_{r}(t)\right\}
\end{aligned}
$$

Then, we replace the term $\left\{B_{r}^{-1}(t)\left[A_{n l}(X, \dot{X}, t)-\bar{\gamma}_{v}\right] d t+d \hat{W}_{r}(t)\right\}$ with $d \bar{W}_{r}(t)$. Therefore, the linearized SDEs in $\left(\widetilde{\bar{X}}_{1}, \widetilde{\bar{X}}_{2}\right)$ are obtainable as

$$
d \tilde{\bar{X}}=\tilde{A} \tilde{\bar{X}} d t+\bar{F}(t) d t+\bar{\gamma}_{v} d t+\sum_{r=1}^{q} B_{r}(t) d \bar{W}_{r}(t)
$$

where, $\bar{\gamma}_{v}(t) \in \mathbf{R}^{2 n}$ is obtained from the normalized forcing vector $\gamma_{v}=\Sigma_{i=1}^{v} S_{i} ; \gamma_{v} \in \mathbf{R}^{n}, v \in \mathbf{Z}^{+}$(just as $\bar{F}(t) \in \mathbf{R}^{2 n}$ is obtainable from the normalized forcing vector $\hat{F}(t) \in \mathbf{R}^{n}$ ) by augmenting the latter with zeros at locations of the displacement components. The Radon-Nikodym derivative is given by

$$
\begin{aligned}
y(t)= & y_{i-1} \exp \left\{\int_{t_{i-1}}^{t} \sum_{j=1}^{n} \sum_{r=1}^{q}\left[b_{r}^{(j)}(t)\right]^{-1}\left[a_{n l}^{(j)}\left(\tilde{\bar{X}}_{1}, \widetilde{\bar{X}}_{2}, t\right)-\gamma_{v}^{(j)}\right] d \bar{W}_{r}(s)\right. \\
& \left.-\frac{1}{2} \int_{t_{i-1}}^{t}\left[b_{r}^{(j)}(t)\right]^{-2}\left[a_{n l}^{(j)}\left(\tilde{\bar{X}}_{1}, \tilde{\bar{X}}_{2}, t\right)-\gamma_{v}^{(j)}\right]^{2} d s\right\}
\end{aligned}
$$

From limited numerical experiments, it has been observed that it generally suffices to use only the first element $S_{1}$ of the subtracting set and thus just employ $\bar{\gamma}_{1}$ as the corrective forcing vector to construct the linearized SDEs (Eq. (13)). We may consequently expect an adequate reduction in the spurious oscillations of $y(t)$ in the process. Figure 2 shows the variation of stochastic process $y(t)$ with and without application of $\gamma_{1}$. As is indeed verifiable from this figure, by using $\gamma_{v}$ (Eq. (14)) we are in fact trying to force the expectation of $y(t)$ close to 1.0. Subsequently, the functional $\nu$ of Eq. (6) can be approximately computed through its Monte Carlo estimator

$$
\hat{v}(t)=\frac{1}{N} \sum_{i=1}^{N} \mathrm{y}^{(i)}(t)\left[f\left(\tilde{X}^{(i)}, \dot{\tilde{X}}^{(i)}, t\right)\right] ; \quad t \in T_{i}
$$

We capture the essence of the idea behind the GLM through the following theorem.
Theorem on Girsanov Linearization. Consider the system of SDEs (Eq. (2)) in terms of the process $\bar{X}(t): \mathbf{R} \rightarrow \mathbf{R}^{2 n}$ with $\bar{X}(t$ $=0)=\bar{X}_{0}$ and $W_{r}(t)$ being a q-dimensional Weiner process under the filtration $F_{t}$. Let the velocity drift vector $A(\bar{X}, t)$ be decomposed additively as $A=A_{l}+A_{n l}$, where $A_{l}$ and $A_{n l}$ are respectively linear and nonlinear in $\bar{X}$. Now consider the linearized SDEs (Eq. (13)) in $\tilde{\bar{X}} \in \mathbf{R}^{2 n}$ with the initial condition $\tilde{\bar{X}}(t=0)=\bar{X}_{0}$. Under $a$ local form of Novikov restriction for $t \in T_{i}=\left(t_{i-1}, t_{i}\right]$ (i.e., $\left.\mathbf{E}\left[\exp \left(\frac{1}{2} \int_{t_{i-1}}^{t}\left(A_{n l}-\bar{\gamma}_{v}\right)^{2} d s\right)\right]<\infty\right)$, the Radon-Nikodym derivative $y(t)$ in Eq. (14) is a positive martingale with respect to $F_{t}$ and measure $P$. Then the measure $\widetilde{Q}$ for the linearized process $\widetilde{\bar{X}}$ is given by $d \tilde{Q}=y(t) d P$ so that the $\tilde{Q}$-law of $\tilde{\bar{X}}$ is same as the P-law of $\bar{X}$. Thus, if $f(\bar{X}, t): \mathbf{R}^{2 n} \times \mathbf{R} \rightarrow \mathbf{R}$ is a (weakly) bounded and measurable function, then the following identity holds: $\mathbf{E}_{P}[f(\bar{X}, t)]$ $=\mathbf{E}_{\tilde{Q}}[y(t) f(\widetilde{\bar{X}}, t)]$.

An "Order Infinity" Algorithm. We have thus far been focusing only on issues related to how to check large oscillations in $y(t)$ and in the process preventing overflows and underflows. However, use of Ito-Taylor expansions for the evaluation of $y(t)$ still remains a stumbling block in a versatile and efficient algorithmic implementation of GLM. Computing MSIs not only requires substantial computational effort, the associated Ito-Taylor expansion also demands that the nonlinear part of the vector field be sufficiently differentiable - a condition that is generally not met in impact and elastoplastic problems. Even though the GLM is a numeric-analytical technique, its implementation as outlined above requires the evaluation of a large number of MSIs for computing $y(t)$ over every time step (see the Appendix). For instance, an $O\left(h^{4}\right)$ time-marching algorithm (map) based on a direct ItoTaylor expansion for an SDOF Duffing oscillator under an additive white noise requires $5 \mathrm{MSIs}$ as compared to $10 \mathrm{MSIs}$ required in the GLM. Indeed, adopting the above form of the GLM requires about twice the number of Ito integrals than that required for solutions of SDEs through a direct Ito-Taylor expansion. Worse, we cannot use such expansions for nondifferentiable nonlinear functions appearing in the original SDEs that are not sufficiently differentiable.

We now propose an alternative procedure for computing $y(t)$ without taking recourse to stochastic Taylor expansion. In particular, we focus on a different procedure for the evaluation of integrals of the forms (within a subinterval $T_{i}$ ) $\int_{t_{i-1}}^{t_{i}} a_{n l}^{(j)}\left(\tilde{\bar{X}}_{1}, \tilde{\bar{X}}_{2}, t\right) d \bar{W}_{r}(s)$ and $\int_{t_{i-1}}^{t_{i}}\left[a_{n l}^{(j)}\left(\tilde{\bar{X}}_{1}, \tilde{\bar{X}}_{2}, t\right)\right]^{2} d s$. Recall that computation of $y(t)$ (Eq. (14)) involves only these two typical integrals. The first may be interpreted as a zero-mean Gaussian random variable with variance $\int_{t_{i-1}}^{t_{i}} \mathbf{E}\left[a_{n l}^{(j)}\left(\tilde{\bar{X}}_{1}, \widetilde{\bar{X}}_{2}, t\right)\right]^{2} d s$, i.e., the integral of the second type. It therefore suffices to restrict our attention to the latter integral alone. We use a Monte Carlo approach for evaluation of this integral [32]. Thus, we treat $\int_{t_{i-1}}^{t_{i}} \mathbf{E}\left[a_{n l}^{(j)}\left(\tilde{\bar{X}}_{1}, \tilde{\bar{X}}_{2}, t\right)\right]^{2} d s$ as a mathematical expectation with respect to a new probability measure $\bar{P}$, whose density is uniformly distributed in $\left[t_{i-1}, t_{i}\right]$. In other words, we have the identity

$$
\int_{t_{i-1}}^{t_{i}} \mathbf{E}\left[a_{n l}^{(j)}\left(\tilde{\bar{X}}_{1}, \tilde{\bar{X}}_{2}, t\right)\right]^{2} d s=h_{i} \mathbf{E}_{P}^{-}\left\{\mathbf{E}\left[a_{n l}^{(j)}\left(\tilde{\bar{X}}_{1}, \tilde{\bar{X}}_{2}, t\right)\right]^{2}\right\}
$$

where the expectation inside the bracket is with respect to the Girsanov-transformed measure $Q$. Accordingly, we generate a set of random time instants $\left\{t\left(\omega_{k}\right) \in T_{i} \mid k \in Z^{+}\right\}$that are uniformly distributed in $T_{i}$. Thus, based on the known analytical solution of the linearized SDEs (Eq. (13)), we can evaluate $\mathbf{E}\left\{\left[a_{n l}^{(j)}\left(\tilde{\bar{X}}_{1}, \tilde{\bar{X}}_{2}, t\right)\right]^{2}\right\}$ as 
a continuous function of $t \in T_{i}$ in closed form and then find its values at each of $t\left(\omega_{k}\right)$. Now, to evaluate the expectation on the right-hand side of Eq. (15), we again employ the Monte Carlo estimator

$\mathbf{E}_{P}^{-}\left\{\mathbf{E}\left[a_{n l}^{(j)}\left(\tilde{\bar{X}}_{1}, \tilde{\bar{X}}_{2}, t\right)\right]^{2}\right\} \cong \frac{1}{N} \sum_{k=1}^{N}\left[\mathbf{E}\left(a_{n l}^{(j)}\left\{\tilde{\bar{X}}_{1}\left[t\left(\omega_{k}\right)\right], \widetilde{\bar{X}}_{2}\left[t\left(\omega_{k}\right)\right], t\left(\omega_{k}\right)\right\}\right)^{2}\right]$

Given the finite precision arithmetic used in all computing machines, it is generally not feasible to increase the accuracy of numerical results indefinitely. For instance, if $10^{-16}$ is the lowest absolute value of a real number that a computer can use in (floating point) algebraic operations, even a so-called " $O\left(h^{\infty}\right)$ method" would only be able to achieve an approximation accuracy of order $<10^{-16}$. If we denote the local order of accuracy in computing the left-hand side of Eq. (16) to be $O\left(h_{i}^{\alpha+1}\right)$, then this is the highest possible order if the identity $h^{\alpha+1}=10^{-16}$ holds. Solving this identity for $\alpha$ and assuming $\mathbf{E}\left\{\left[a_{n l}^{(j)}\left(\tilde{\bar{X}}_{1}, \widetilde{\bar{X}}_{2}, t\right)\right]^{2}\right\}$ to be sufficiently smooth, it is possible to represent this expectation locally over $T_{i}$ in terms of an interpolating polynomial of order $\alpha$ with $\alpha+1$ interpolation points in $T_{i}$. If such an arrangement is adopted, then one may compute $\mathbf{E}\left\{\left[a_{n l}^{(j)}\left(\tilde{\bar{X}}_{1}, \tilde{\bar{X}}_{2}, t\right)\right]^{2}\right\}$ anywhere in $T_{i}$ based on its known discrete values at just $\alpha+1$ points. Such an arrangement makes sense as the determination of $\mathbf{E}\left\{\left[a_{n l}^{(j)}\left(\widetilde{\bar{X}}_{1}, \widetilde{\bar{X}}_{2}, t\right)\right]^{2}\right\}$ from the known linearized solution is quite computationally intensive. However, in order to avoid numerical instabilities (such as Runge's phenomenon [33]) associated with Lagrangian interpolating polynomials, we prescribe using spline interpolations of the appropriate order. Thus, we subdiscretize the interval $T_{i}$ into $\alpha$ subintervals (of equal lengths) with $(\alpha+1)$ equispaced points ( $t_{i-1}$ and $t_{i}$ being two boundary points), obtain the solution $\mathbf{E}\left\{\left[a_{n l}^{(j)}\left(\tilde{\bar{X}}_{1}, \tilde{\bar{X}}_{2}, t\right)\right]^{2}\right\}$ only at those points and pass the spline interpolant through these points.

\section{Numerical Illustrations}

For purposes of illustration, we presently choose two SDOF and one 2-DOF nonlinear oscillators with cubic nonlinearity. The two SDOF oscillators are the hardening Duffing (HD) and the two-well Duffing-Holmes (DH) oscillators. Taken together, these two SDOF oscillators exhibit a large class of phenomena characteristic of many higher-dimensional nonlinear dynamical systems. The purpose behind choosing the 2-DOF example is to demonstrate the ready applicability of the GLM to nonlinear MDOF problems.

An SDOF Hardening Duffing Oscillator (HD) Under Additive Noise. First consider an HD oscillator only under additive white noise. The governing equation is of the form

$$
\ddot{x}+C \dot{x}+K_{1} x+K_{2} x^{3}=\sigma \dot{W}(t)
$$

where $K_{1}, K_{2}$, and $C$ are positive real constants representing the stiffness, the "strength" of nonlinearity, and the coefficient of viscous damping, respectively, $W(t)$ is a standard Brownian motion process, and $\sigma$ denotes the intensity of noise. Equation (18) is subject to the initial condition $X_{0}=\left\{x_{0}, \dot{x}_{0}\right\}^{T}$. We also denote $X$ $:=\left\{x_{1}, x_{2}\right\}^{T}$. Writing Eq. (18) in the Girsanov-transformed form of Eq. (7), the linearized SDEs and the scalar SDE corresponding to the exponential correction process over $T_{i}$ may be written as

$$
\begin{aligned}
& d \tilde{x}_{1}(t)=\widetilde{x}_{2}(t) d t \\
& d \widetilde{x}_{2}(t)=\left(-C \tilde{x}_{2}-K_{1} \widetilde{x}_{1}\right) d t+\underbrace{\sigma\left\{\sigma^{-1} K_{2} \tilde{x}_{1}^{3} d t+d W(t)\right\}}_{d \hat{W}(t)} \\
& d y(t)=-\sigma^{-1} K_{2} \tilde{x}_{1}^{3} y d \hat{W}(t)
\end{aligned}
$$

Solutions for the linearized state $\tilde{X}:=\left\{\tilde{x}_{1}, \tilde{x}_{2}\right\}^{T}$ and the correction process $y(t)$ presently take the form

$$
\begin{aligned}
\tilde{X}(t)= & \exp \tilde{A}\left(t-t_{i-1}\right) X_{0}+\exp \tilde{A}\left(t-t_{i-1}\right) \int_{t_{i-1}}^{t} \exp \left(-\tilde{A}\left(s-t_{i-1}\right)\right. \\
& \times\left[\begin{array}{ll}
0 & 0 \\
0 & \sigma
\end{array}\right]\left\{\begin{array}{c}
0 \\
d \hat{W}_{1}(\dot{s})
\end{array}\right\} \\
y(t)= & \exp \left[\int_{t_{i-1}}^{t}\left(-\sigma^{-1} K_{2} \tilde{x}_{1}^{3}\right) d \hat{W}(t)-\frac{1}{2} \int_{t_{i-1}}^{t}\left(-\sigma^{-1} K_{2} \widetilde{x}_{1}^{3}\right)^{2} d s\right]
\end{aligned}
$$

where $\tilde{A}=\left[\begin{array}{cc}0 & 1 \\ -K_{1}-C\end{array}\right]$ and $B_{r}(t)=\left\{\begin{array}{l}0 \\ \sigma\end{array}\right\}$. The exponential solution for $y(t)$ has integral arguments that may be expanded in terms of MSIs through the Ito-Taylor expansion (see Appendix). By using the estimator Eq. (15) we may readily obtain the expectation of a given function $f(X, t)$.

Now we turn attention to the linearization method using the subtracting set $S^{i}$ corresponding to the Ito-Taylor expansion of the nonlinear vector function $K_{2} x_{1}^{3}$. Consider first element $S_{1}^{i}=$ $-K_{2} x_{1, i-1}^{3}$ of $S^{i}$ and add it to the linear part of the drift $a_{l}$ while subtracting the same from the nonlinear part $a_{n l}$. The modified drift term in the linearized velocity equation is then $a_{l}(\tilde{X}, t)=$ $-C \tilde{x}_{2}-K_{1} \tilde{x}_{1}-K_{2} \tilde{x}_{1, i-1}^{3}$. The term that goes into the transformed Brownian motion is $a_{n l}(\tilde{X}, t)=-K_{2} x_{1}^{3}+K_{2} \widetilde{x}_{1, i-1}^{3}$. We then have the following incremental form for the Girsanov-linearized SDEs:

$$
\begin{aligned}
d\left\{\begin{array}{l}
\tilde{x}_{1} \\
\tilde{x}_{2}
\end{array}\right\}= & {\left[\begin{array}{cc}
0 & 1 \\
-K_{1} & -C
\end{array}\right] d t+\left\{\begin{array}{c}
0 \\
-K_{2} \widetilde{x}_{1, i-1}^{3}
\end{array}\right\} d t } \\
& +\sigma\{\underbrace{-\sigma^{-1}\left[K_{2} \widetilde{x}_{1}^{3}-K_{2} \widetilde{x}_{1, i-1}^{3}\right] d t+d W(t)}_{d \bar{W}(t)}\}
\end{aligned}
$$

The additional scalar SDE for the correction term is

$$
d y(t)=-\sigma^{-1}\left[K_{2} \widetilde{x}_{1}^{3}-K_{2} \tilde{x}_{1, i-1}^{3}\right] y d \bar{W}(t)
$$

This yields the solution for $y(t)$ as

$$
\begin{aligned}
y(t)= & y_{i-1} \exp \left[\int_{t_{i-1}}^{t}\left(-\sigma^{-1}\left[K_{2} \tilde{x}_{1}^{3}-K_{2} \tilde{x}_{1, i-1}^{3}\right]\right) d \bar{W}(t)\right. \\
& \left.-\frac{1}{2} \int_{t_{i-1}}^{t}\left(-\sigma^{-1}\left[K_{2} \widetilde{x}_{1}^{3}-K_{2} \tilde{x}_{1, i-1}^{3}\right]\right)^{2} d s\right]
\end{aligned}
$$

According to the notation in Eq. (13), we have $\bar{\gamma}=\left\{\begin{array}{c}0 \\ -K_{2} x_{1, i-1}^{3}\end{array}\right\}$ and $B_{r}(t)=\left\{\begin{array}{c}0 \\ \sigma\end{array}\right\}$. Note that both the integrals in the right-hand side of Eq. (23) are stochastic and computed using both the versions of the GLM as described earlier. Version 1 (henceforth referred to as GLM-I), which uses an Ito-Taylor expansion, is presently done to $O\left(h^{4}\right)$ and the modeling details of the associated MSIs are described in the Appendix. Recall that, unlike version 1, version 2 (GLM-II) provides a so-called $O\left(h^{\infty}\right)$ solution (i.e., the accuracy of solution is only affected by the finiteness of ensemble sizes) and does not require computing any derivatives and MSIs. 

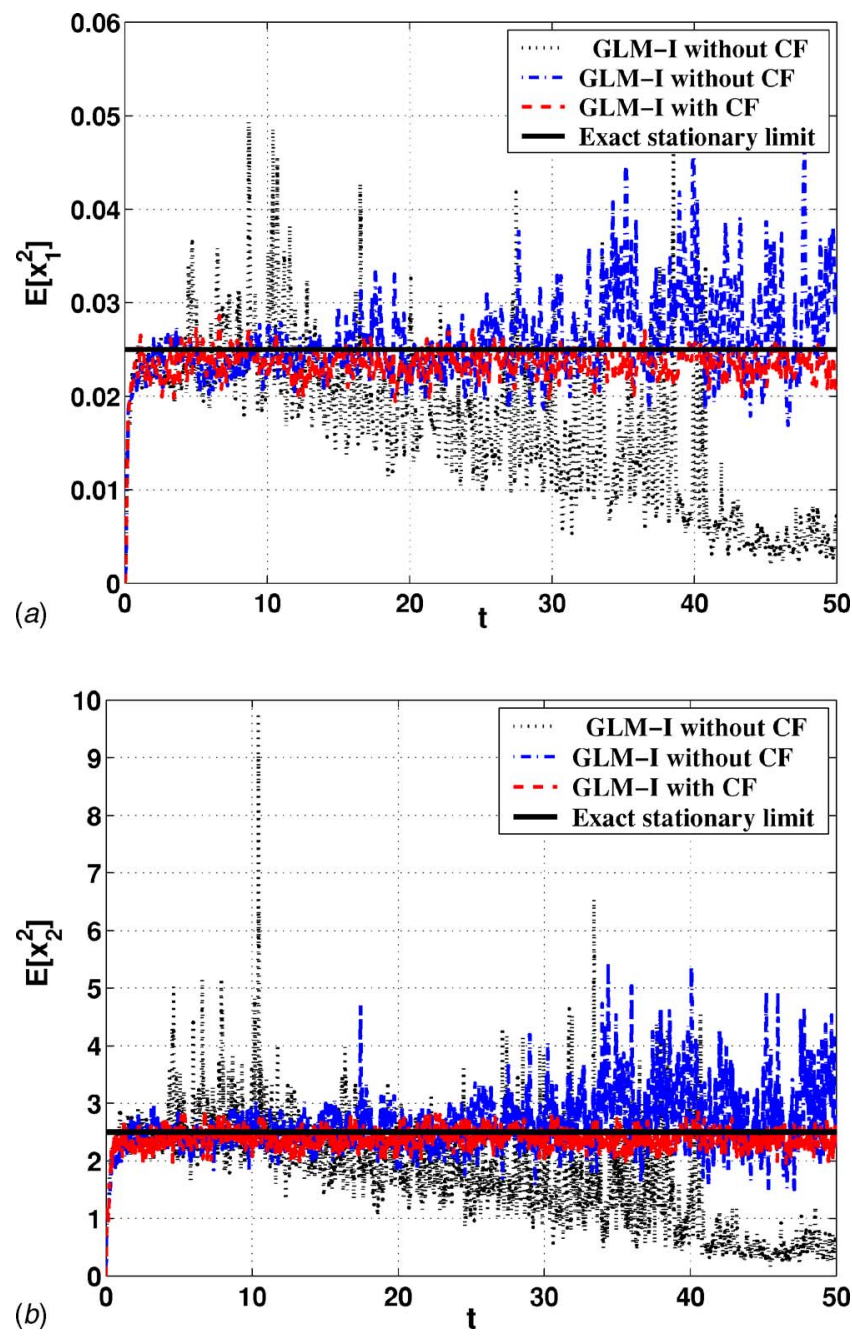

Fig. 3 The HD oscillator (Eq. (18))-histories of second moments: (a) $E\left[x_{1}^{2}\right]$ and (b) $E\left[x_{2}^{2}\right] ; C=5.0, K_{1}=K_{2}=100.0, \sigma=5.0, h$ $=0.01, X_{0}=\{0,0\}^{T}$; solid black lines indicate exact stationary limits

In Figs. 3(a) and 3(b), we show time histories of $E\left(x_{1}^{2}\right)$ and $E\left(x_{2}^{2}\right)$ with the two variants of GLM-I (with and without the first element $S_{1}^{i}$ of the subtracting set leading to the corrective forcing vector $\left.\bar{\gamma}_{1}\right)$ as well as the exact stationary limits. Note that the exact stationary (joint) density function is available in this case through the solution of the reduced Fokker-Planck equation and is given by [34]

$$
p(x, \dot{x})=R \exp \left(\frac{-C \dot{x}^{2}}{\sigma^{2}}-\int_{0}^{x} C \frac{K_{1} q+K_{2} q^{3}}{0.5 \sigma^{2}} d q\right)
$$

where the real constant $R$ has to be so found as to satisfy the normalization constraint $\iint_{-\infty}^{\infty} p(x, \dot{x}) d x d \dot{x}=1$. The statistical moment of any deterministic function $\Phi(x, \dot{x})$ may then be found as $E[\Phi(x, \dot{x})]=\iint_{-\infty}^{\infty} \Phi(x, \dot{x}) p(x, \dot{x}) d x d \dot{x}$. The integrations required to find the stationary limits are presently computed using the symbolic manipulator MAPLE®. In Fig. 3, we see two instances of solutions via GLM-I without the usage of the corrective forcing $(\mathrm{CF})$. While, in one such instance, we have an overflowing trend, the other instance has a typical solution that tends to underflow. The GLM-I with the CF however appears to work without numerical instabilities, and the resulting solutions match well the exact stationary limit as time progresses. Thus, unless otherwise specified, we apply GLM-I and II with the CF from now onwards.
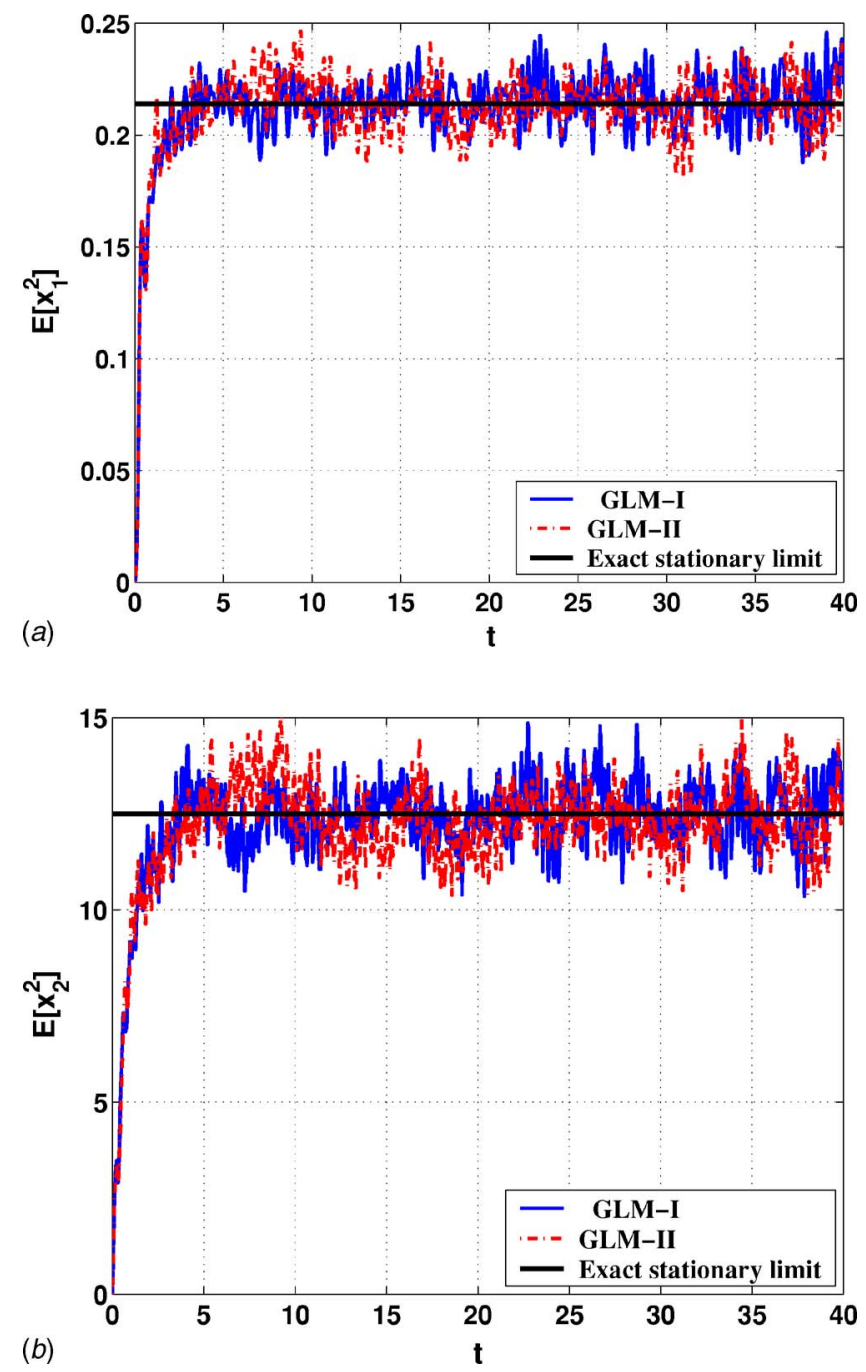

Fig. 4 The HD oscillator (Eq. (18))-histories of second moments through GLM-I and II: (a) $\mathrm{E}\left[x_{1}^{2}\right]$ and (b) $\mathrm{E}\left[x_{2}^{2}\right] ; C=1.0, K_{1}$ $=10.0, K_{2}=100.0, \sigma=5.0, h=0.01, X_{0}=\{0,0\}^{T}$; solid black lines indicate exact stationary limits

Figure 4 shows second moment histories of displacement and velocity components via GLM-I and II when the nonlinearity parameter $\left(K_{2}\right)$ is ten times the stiffness $\left(K_{1}\right)$ and the additive noise intensity $(\sigma=5.0)$ is very high. Also shown in Fig. 4 are the corresponding stationary limits. In this and many other such cases, both the versions of GLM work accurately, even though variance of the estimate appears to be less with GLM-II for the same ensemble size. Thus, in all such cases, results would henceforth be plotted with any one of the versions. It is interesting to observe (see Eq. (24)) that $K_{2}$ only affects the stationary limit for $E\left[x_{1}^{2}(t)\right]$ but not that for $E\left[x_{2}^{2}(t)\right]$. Figure 5 shows the variations of $E\left[x_{1}^{2}(t)\right]$, as obtained through GLM-I, for different values of $K_{2}$ as well as the associated stationary limits (black and solid lines). Time histories of $E\left[x_{1}^{2}(t)\right]$ are plotted in Fig. 6 using GLM-II for different values of the time step-size, $h$. Incidentally, for $h=0.05$, GLM-I fails to work (as indicated in the inset) and numerical instabilities set in for $t \geqslant 0.8 \mathrm{~s}$ or so.

An HD Oscillator Under Combined Deterministic Excitation and Additive Noise. The governing equation is presently taken to be in the form [9] 


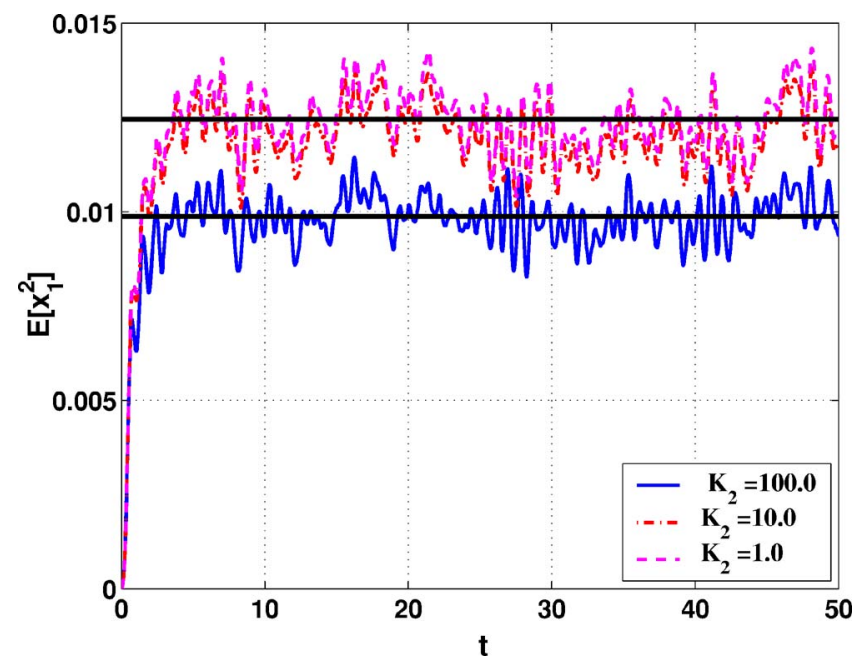

Fig. 5 The HD oscillator (Eq. (18)) $-E\left[x_{1}^{2}\right]$ for different values of $K_{2}$ using GLM-II; $C=1.0, \quad K_{1}=10.0, \quad \sigma=0.50, h=0.01, \quad X_{0}$ $=\{0,0\}^{T}$; solid black lines indicate exact stationary limits

$$
\ddot{x}+2 \pi \varepsilon_{1} \dot{x}+4 \pi^{2} \varepsilon_{2} x\left(1+x^{2}\right)=4 \pi^{2} \varepsilon_{3} \cos (2 \pi t)+4 \pi^{2} \varepsilon_{4} \dot{W}_{1}(t)
$$

A look at the solution for $y(t)$ (see Eq. (13)) shows that it contains the inverse of the diffusion coefficient (noise intensity). Since division by a small number could cause numerical problems, it would be interesting to see if the GLM had any instability for very low noise intensity. Figure 7 shows plots of variance histories of displacement and via GLM-II corresponding to the HD oscillator of Eq. (25) with $\varepsilon_{4}=0.0005$ (i.e., very low noise intensity). Here, the sinusoidal forcing parameter $\varepsilon_{3}=1.0$ is so chosen that the oscillator exhibit a dumb-bell-shaped one-periodic solution for $\varepsilon_{4}$ $=0$ and such is structure is expected to be preserved under "small" additive noise. Since no exact solutions are presently available, we choose the implicit stochastic Newmark method (SNM) [35,36] and the explicit stochastic Heun method (SHS) [5] for purposes of comparison. Although the displacement updates via SNM and SHS are comparable, the velocity update via SNM is

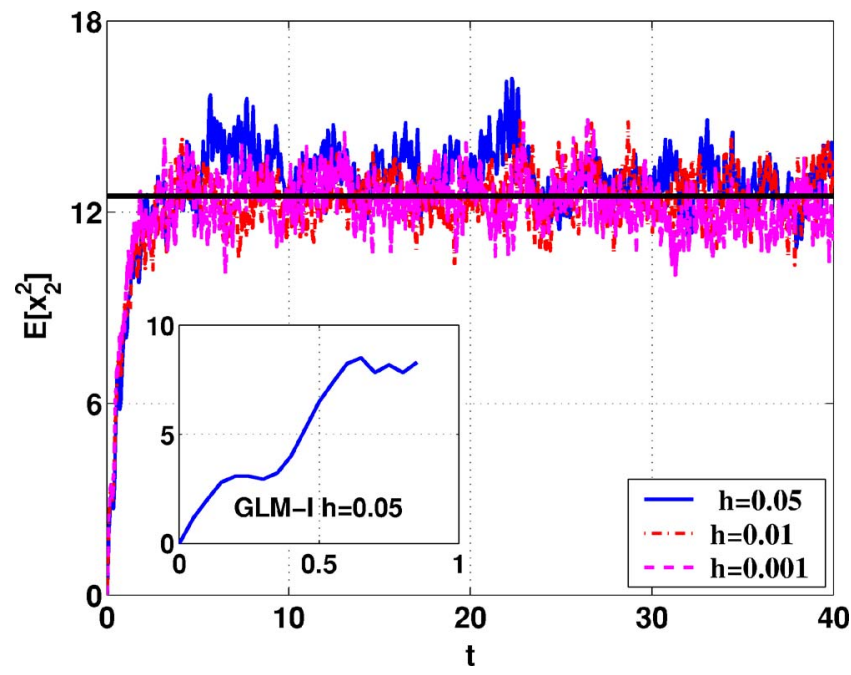

Fig. 6 The HD oscillator (Eq. (18))-histories of $E\left[x_{2}^{2}\right]$ for different values of $h$ using GLM-II; $C=1.0, K_{1}=10.0, K_{2}=100.0$, $F(t)=0, \sigma=5.0, X_{0}=\{0,0\}^{T}$; the solid black line indicates the exact stationary limit. Inset shows that GLM-I terminates unsuccessfully before $1 \mathrm{~s}$ for $h=0.05$.
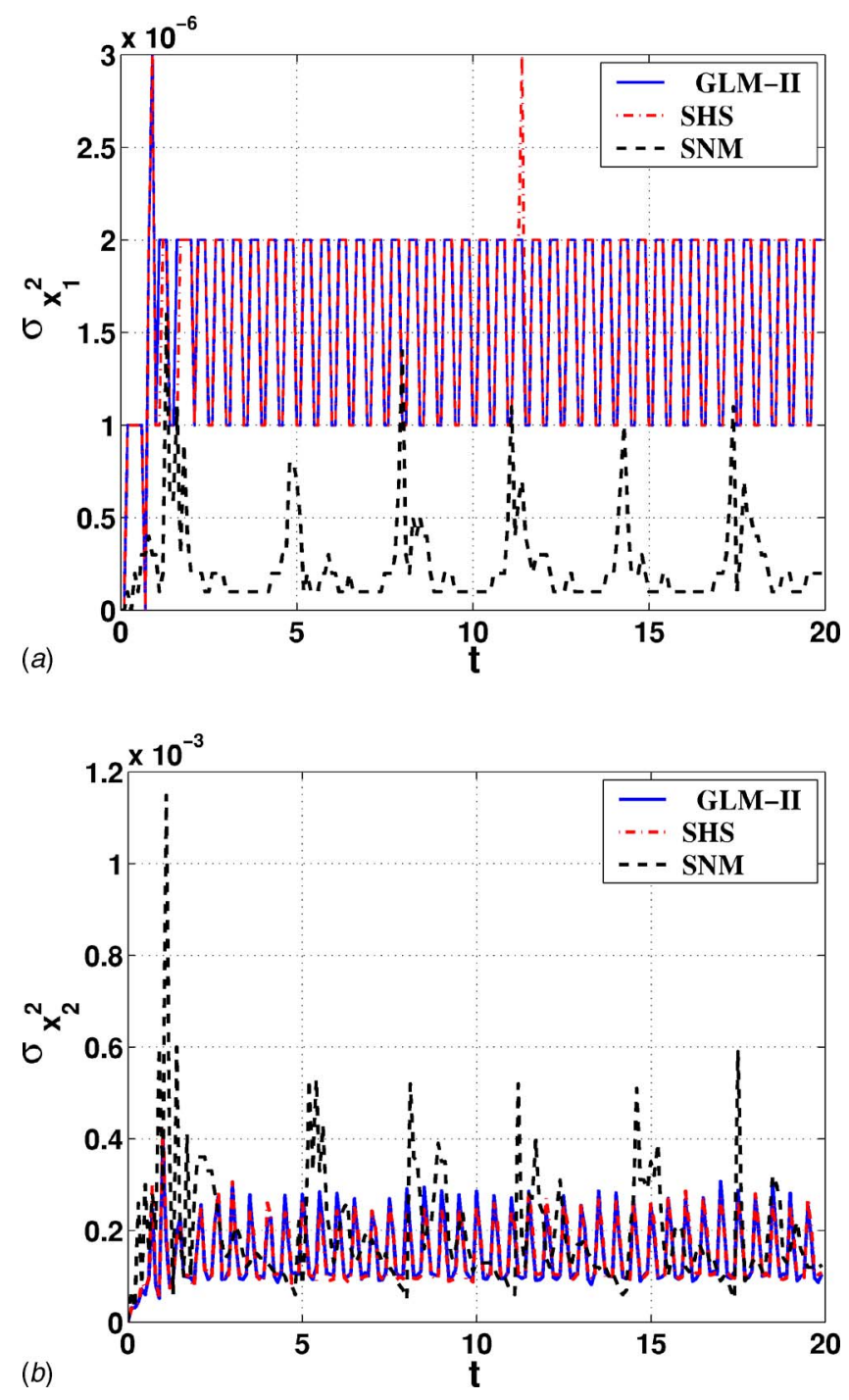

Fig. 7 The HD oscillator (Eq. (25)) $-\varepsilon_{1}=0.25, \varepsilon_{2}=1.0, \varepsilon_{3}=1.0$, $\varepsilon_{4}=0.0005, h=0.01, X_{0}=\{0,0\}^{T}$; histories of (a) variance of displacement and (b) variance of velocity, using GLM-II, SHS, and SNM

just as good as a drift-implicit Euler method, which is worse than the SHS. As is seen from Fig. 7, while solutions via GLM and SHS match reasonably, solutions via the SNM are too far off. However, over most of the parameter ranges, the results through SNM indeed match closely with those via GLM. A typical such case is reported in Fig. 8.

A Duffing-Holmes (DH) Oscillator Under Combined Deterministic Excitation and Additive Noise. We now take up a DH oscillator subject to a sinusoidal force and an additive white-noise excitation. For $\varepsilon_{3}=\varepsilon_{4}=0$, the system has two potential wells (basins of attraction) corresponding to the two stable fixed points at $(x, \dot{x})=(1,0)$ and $(x, \dot{x})=(-1,0)$. This oscillator may, for instance, be a useful model for the nonlinear dynamics of a periodically forced buckled beam. The governing equation is

$$
\ddot{x}+2 \pi \varepsilon_{1} \dot{x}+4 \pi^{2} \varepsilon_{2} x\left(-1+x^{2}\right)=4 \pi^{2} \varepsilon_{3} \cos (2 \pi t)+4 \pi^{2} \varepsilon_{4} \dot{W}_{1}(t)
$$

In the absence of the diffusion term, this oscillator exhibits a wide variety of multiperiodic and chaotic solutions. Figure 9 shows displacement variance histories as well as phase plots of $E\left[x_{1}(t)\right]$ versus $E\left[x_{2}(t)\right]$ as determined via GLM-I, SHS, and SNM. The 

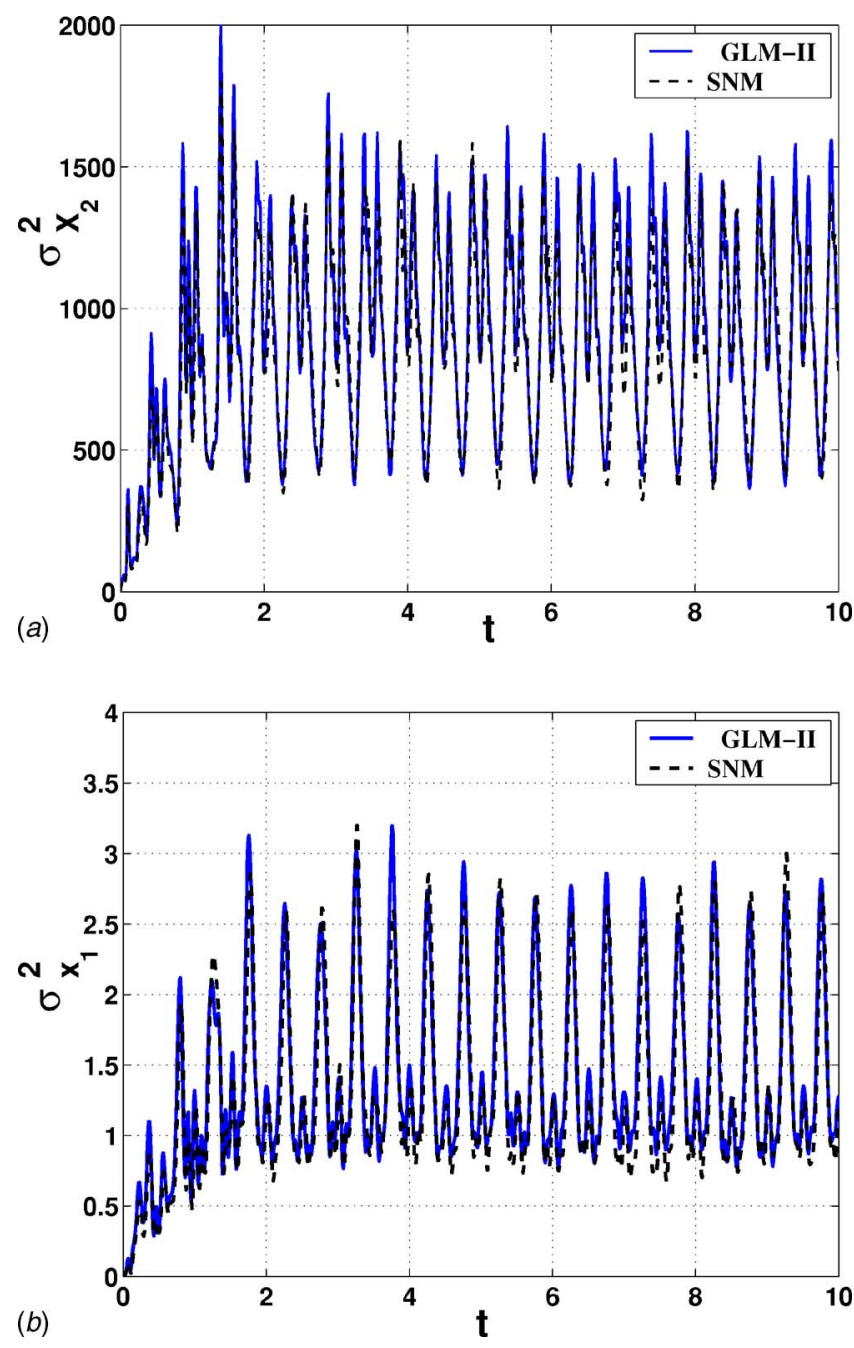

Fig. 8 The HD oscillator (Eq. (25)) -histories of (a) variance of displacement and $(b)$ variance of velocity using GLM-II and SNM; $\varepsilon_{1}=0.25, \varepsilon_{2}=1.0, \varepsilon_{3}=42.0, \varepsilon_{4}=1.0, h=0.01, X_{0}=\{0,0\}^{\top}$

parameters $\varepsilon_{1}, \varepsilon_{2}, \varepsilon_{3}$ are so chosen that they belong to the chaotic regime of the oscillator when $\varepsilon_{4}=0$. When the noise intensity is small (presently $\varepsilon_{4}=0.05$ ), the SNM again fails to work accurately with SHS and GLM still providing a close match (Fig. 9). However, as $\varepsilon_{4}$ becomes higher, SNM-based results match closely with those via GLM and SHS (Fig. 10).

A 2-DOF Nonlinear Oscillator Under Additive Noises. In order to numerically demonstrate the applicability of the GLM to higher dimensional oscillators, we choose a 2-DOF nonlinear oscillator driven by additive white noises and described by

$$
\begin{gathered}
\ddot{x}_{1}+C_{1} \dot{x}_{1}+\left(K_{1}+K_{2}\right) x_{1}-K_{2} x_{3}+\alpha x_{1}^{3}=\sigma_{1} \dot{W}_{1}(t) \\
\ddot{x}_{3}+C_{2} \dot{x}_{3}+\left(K_{2}+K_{3}\right) x_{3}-K_{2} x_{1}=\sigma_{2} \dot{W}_{2}(t)
\end{gathered}
$$

The derivation of the GLM for this oscillator follows precisely from the general formulation recorded in the previous section. However, for completeness, we retrace the main steps briefly. Thus, introducing the state space vector $\bar{X}:=\left\{x_{1}^{(1)}, x_{2}^{(1)}, x_{1}^{(2)}, x_{2}^{(2)}\right\}^{T}$, the displacement vector $X_{1}:=\left\{x_{1}^{(1)}, x_{1}^{(2)}\right\}^{T}$ and the velocity vector $X_{2}:=\left\{x_{2}^{(1)}, x_{2}^{(2)}\right\}^{T}$, the incremental form of the above SDE-s is written as

$$
d x_{1}^{(1)}=x_{2}^{(1)} d t
$$
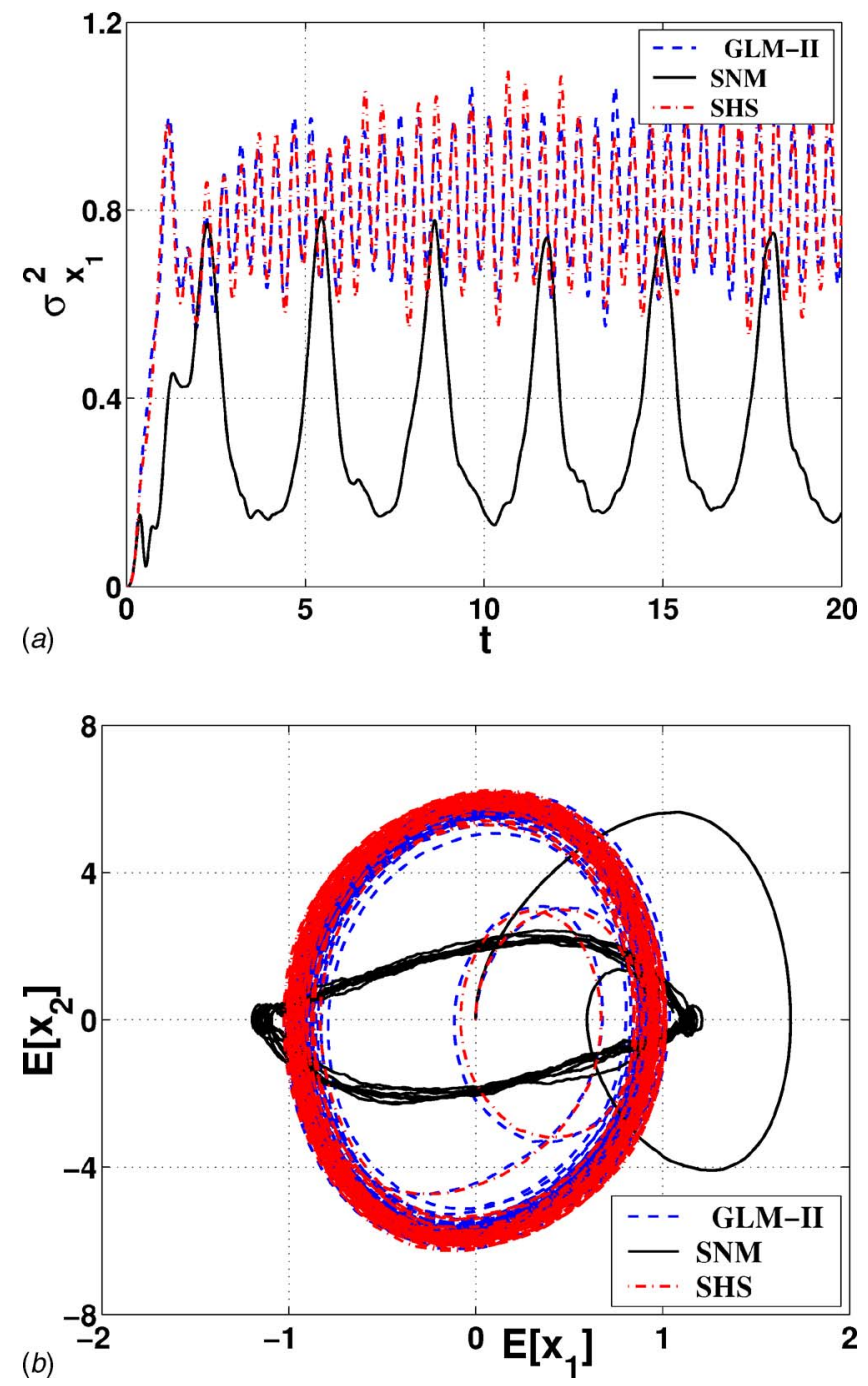

Fig. 9 The DH oscillator (Eq. (26)) -histories of (a) variance of displacement and $(b)$ phase plot of $\mathrm{E}\left[x_{2}\right]$ and $\mathrm{E}\left[x_{1}\right]$ using GLMII, SNM and SHS; $\varepsilon_{1}=0.25, \varepsilon_{2}=0.5, \varepsilon_{3}=0.5, \varepsilon_{4}=0.10, h=0.01, x_{0}$ $=\{0,0\}^{T}$

$$
\begin{gathered}
d x_{2}^{(1)}=\left[-C_{1} x_{2}^{(1)}-\left(K_{1}+K_{2}\right) x_{1}^{(1)}-\alpha\left(x_{1}^{(1)}\right)^{3}+K_{2} x_{1}^{(2)}\right] d t+\sigma_{1} d W_{1}(t) \\
d x_{1}^{(2)}=x_{2}^{(2)} d t \\
d x_{2}^{(2)}=\left[-C_{2} x_{2}^{(2)}-\left(K_{2}+K_{3}\right) x_{1}^{(2)}+K_{2} x_{1}^{(1)}\right] d t+\sigma_{2} d W_{2}(t)
\end{gathered}
$$

These SDEs are subject to the initial condition vector $\bar{X}_{0}$ $=\left\{x_{10}^{(1)}, x_{20}^{(1)}, x_{10}^{(2)}, x_{20}^{(2)}\right\}^{T}$. Now in order to derive the GLM-based linearized form (as in Eq. (13)) over the $i$ th interval $T_{i}$, we need to construct the corrective forcing $\bar{\gamma}_{1}^{(i)}$ by using the first element $S_{1}^{i}$ of the subtracting set $S^{i}$. Accordingly, the linearized and residual (nonlinear) parts of the velocity drift corresponding to the second scalar SDE in Eq. (28) are given by

$$
\begin{gathered}
a_{l}^{(2)}(\tilde{\bar{X}}, t)=-C_{1} \widetilde{x}_{2}^{(1)}-\left(K_{1}+K_{2}\right) \widetilde{x}_{1}^{(1)}+K_{2} \widetilde{x}_{1}^{(2)}-\alpha\left(\widetilde{x}_{1, i-1}^{(1)}\right)^{3} \\
\text { and } a_{n l}^{(2)}(\tilde{\bar{X}}, t)=-\alpha\left(\widetilde{x}_{1}^{(1)}\right)^{3}+\alpha\left(\widetilde{x}_{1, i-1}^{(1)}\right)^{3}
\end{gathered}
$$

Then, the GLM-based linearized SDE-s are given by 


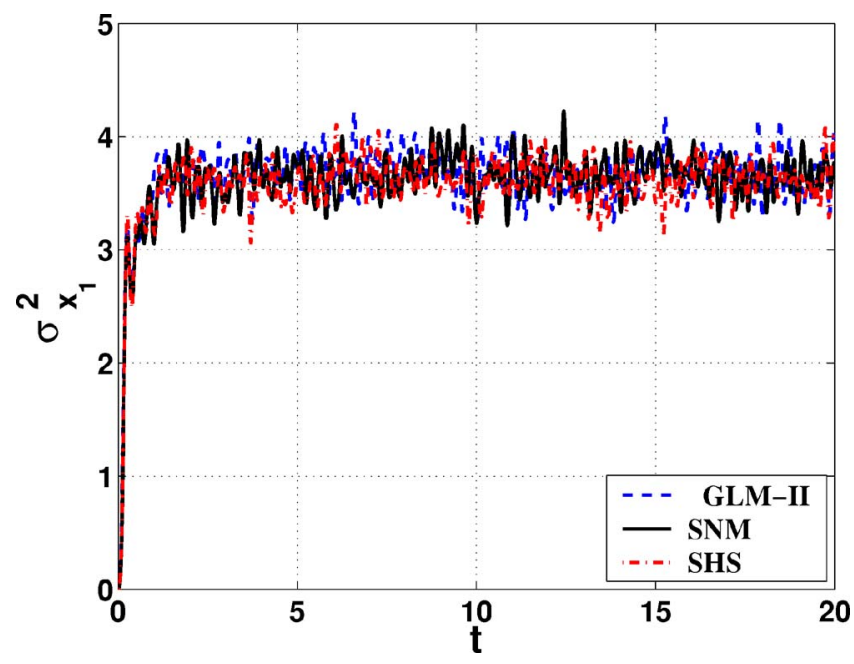

Fig. 10 The DH oscillator (Eq. (26)) -histories of variance of displacement using GLM-II, SNM and SHS; $\varepsilon_{1}=0.25, \varepsilon_{2}=0.5, \varepsilon_{3}$ $=0.5, \varepsilon_{4}=1.0, h=0.01, X_{0}=\{0,0\}^{T}$

$$
\begin{aligned}
d \widetilde{x}_{1}^{(1)}(t)= & \widetilde{x}_{2}^{(1)}(t) d t \\
d \widetilde{x}_{2}^{(1)}(t)= & {\left[-C_{1} \tilde{x}_{2}^{(1)}-\left(K_{1}+K_{2}\right) \tilde{x}_{1}^{(1)}+K_{2} \tilde{x}_{1}^{(2)}-\alpha\left(\tilde{x}_{1, i-1}^{(1)}\right)^{3}\right] d t } \\
& +\sigma_{1} \underbrace{\left.-\sigma_{1}^{-1}\left[\alpha\left(\tilde{x}_{1}^{(1)}\right)^{3}-\alpha\left(\tilde{x}_{1, i-1}^{(1)}\right)^{3}\right] d t+d W_{1}(t)\right\}}_{d \tilde{W}_{1}(t)} \\
d \tilde{x}_{1}^{(2)}(t)= & \tilde{x}_{2}^{(2)}(t) d t \\
d \tilde{x}_{2}^{(2)}(t)= & {\left[-C_{2} \tilde{x}_{2}^{(2)}-\left(K_{2}+K_{3}\right) \tilde{x}_{1}^{(2)}+K_{2} \tilde{x}_{1}^{(1)}\right] d t+\sigma_{2} d \bar{W}_{2}(t) }
\end{aligned}
$$

where $\bar{W}_{2}(t)=W_{2}(t)$. The scalar SDE governing the RadonNikodym derivative is

$$
d y(t)=-\sigma_{1}^{-1}\left[\alpha\left(\widetilde{x}_{1}^{(1)}\right)^{3}-\alpha\left(\widetilde{x}_{1, i-1}^{(1)}\right)^{3}\right] y d \bar{W}_{1}(t)
$$

Solutions for $\tilde{\bar{X}}(t)$ and the Radon-Nikodym derivative turn out to be

$$
\begin{aligned}
\tilde{\bar{X}}= & \exp \left(\tilde{A} \int_{t_{i-1}}^{t} d s\right) \tilde{\bar{X}}_{0}+\exp \left(\tilde{A} \int_{t_{i-1}}^{t} d s\right) \int_{t_{i-1}}^{t} \exp \left(-\tilde{A} \int_{t_{i-1}}^{s} d s_{1}\right) \\
& \times\left[\begin{array}{cccc}
0 & 0 & 0 & 0 \\
0 & -\alpha\left(\widetilde{x}_{1, i-1}(1)\right. & 0 & 0 \\
0 & 0 & 0 & 0 \\
0 & 0 & 0 & 0
\end{array}\right] d s+\exp \left(\tilde{A} \int_{t_{i-1}}^{t} d s\right) \\
& \times \int_{t_{i-1}} \exp \left(-\tilde{A} \int_{t_{i-1}}^{s} d s_{1}\right)\left[\begin{array}{cccc}
0 & 0 & 0 & 0 \\
0 & \sigma_{1} & 0 & 0 \\
0 & 0 & 0 & 0 \\
0 & 0 & 0 & \sigma_{2}
\end{array}\right]\left\{\begin{array}{c}
0 \\
d \bar{W}_{1}(s) \\
0 \\
d \bar{W}_{2}(s)
\end{array}\right\}
\end{aligned}
$$
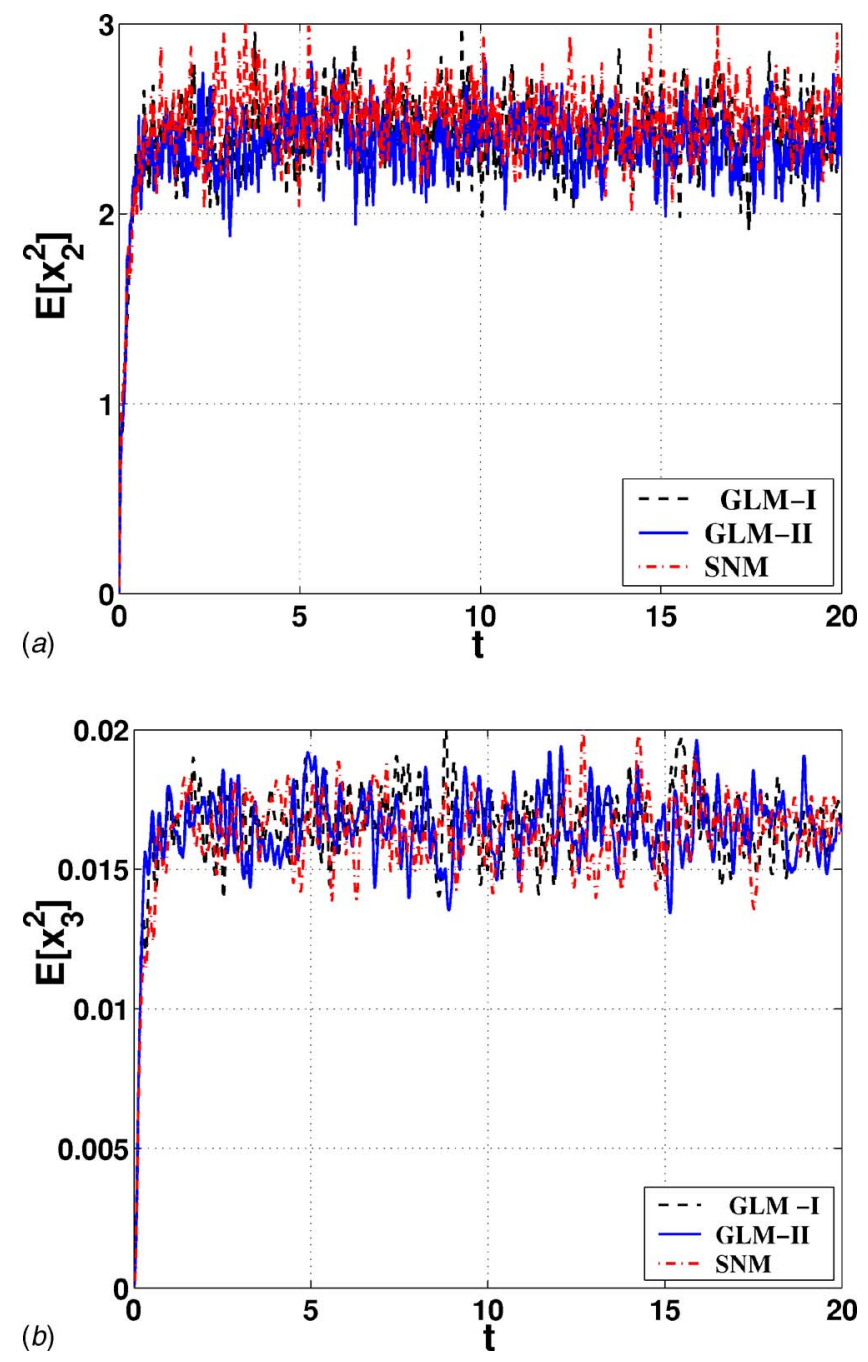

Fig. 11 The 2-DOF oscillator (Eq. (27)) - second moment histories using GLM-I, GLM-II, and SNM: (a) $\mathrm{E}\left[x_{2}^{2}\right]$ and (b) $\mathrm{E}\left[x_{3}^{2}\right]$; $C_{1}=C_{2}=5.0, K_{1}=K_{2}=K_{3}=100.0, \alpha=100.0, \quad \sigma_{1}=\sigma_{2}=5.0, h=0.01$, $\bar{X}_{0}=\{0,0,0,0\}^{T}$

$$
\begin{aligned}
y(t)= & \exp \left(\int_{t_{i-1}}^{t}\left\{-\sigma_{1}^{-1}\left[\alpha\left(\widetilde{x}_{1}^{(1)}\right)^{3}-\alpha\left(\widetilde{x}_{1, i-1}^{(1)}\right)^{3}\right]\right\} d \bar{W}_{1}(s)\right. \\
& \left.-\frac{1}{2} \int_{t_{i-1}}^{t}\left\{\sigma_{1}^{-1}\left[\alpha\left(\widetilde{x}_{1}^{(1)}\right)^{3}-\alpha\left(\widetilde{x}_{1, i-1}^{(1)}\right)^{3}\right]\right\}^{2} d s\right)
\end{aligned}
$$

For $i=1$, we have the initial condition $\tilde{\bar{X}}_{0}=\left\{x_{1,0}^{(1)}, x_{2,0}^{(1)}, x_{1,0}^{(2)}, x_{2,0}^{(2)}\right\}^{T}$ and the system coefficient matrix for the linearized vector SDE is given by

$$
\tilde{A}=\left[\begin{array}{cccc}
0 & 1 & 0 & 0 \\
-\left(K_{1}+K_{2}\right) & -C_{1} & K_{2} & 0 \\
0 & 0 & 0 & 1 \\
K_{2} & 0 & -\left(K_{2}+K_{3}\right) & -C_{2}
\end{array}\right]
$$

Recall that the expectation of a given scalar function $f(\bar{X}, t)$ may be computed as $\mathbf{E}[f(\bar{X}, t)]=\mathbf{E}[y(t) f(\tilde{\bar{X}}, t)]$. 

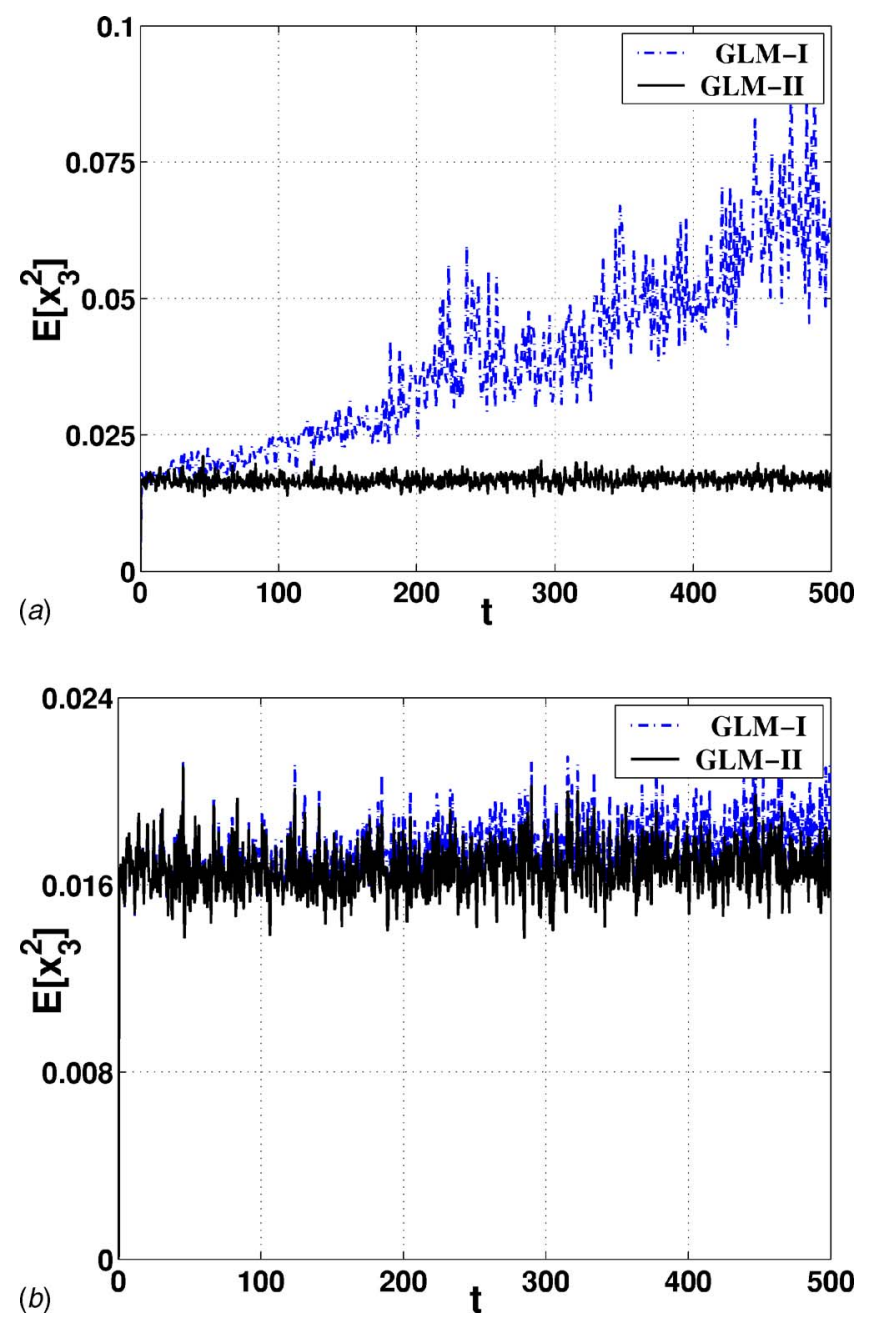

Fig. 12 The 2-DOF oscillator (Eq. (27))-plots of $\mathrm{E}\left[x_{3}^{2}\right]$ using GLM-I and GLM-II for step sizes (a) $h=0.01$ and (b) $h=0.005$; $C_{1}=C_{2}=5.0, \quad K_{1}=K_{2}=K_{3}=100.0, \quad \alpha=100.0, \quad \sigma_{1}=\sigma_{2}=5.0, \quad \bar{X}_{0}$ $=\{0,0,0,0\}^{T}$

Figure 11 shows a couple of second moment histories via GLM-I and GLM-II over $0-20 \mathrm{~s}$ with a step size $h=0.01$. While the closeness of solutions through the two versions of the GLM appears to be impeccable over this interval, the power of GLM-II over GLM-I is brought out if the same histories are plotted over a much longer interval, as in Fig. 12(a). Here, we have used the same step size and plotted the histories of $E\left[x_{3}^{2}(t)\right]$ over $t$ $\in[0,500]$ via the two versions of the GLM. While GLM-II shows no numerical instability, the solution through GLM-I clearly exhibits an overflowing trend and becomes unacceptably inaccurate. However, as shown in Fig. 12(b), GLM-I works well even over such a long interval if the step size is reduced (in this case halved).

\section{Conclusions}

A weak form of linearization method, applicable to nonlinear oscillators under additive stochastic excitations, is developed based on a Girsanov transformation of probability measures. The proposed GLM suffers from no dimensionality curse and has the potential to achieve an accuracy of approximation that is unparalleled by any other existing method to the authors' knowledge. However, a major problem lies with handling the exponential correction term (martingale), which shows high sensitivity to how the linearization is being attempted and often exhibits numerically spurious oscillations. As a result, the GLM-based solution often tends to overflow or underflow as time progresses. In order that such numerical obstacles are virtually removed, a modification is effected in the linearized form and the process of linearization is employed over small intervals of time, as in a numerical method. It is this latter strategy that imparts a numeric-analytic character to the GLM. Two different versions of the GLM are presented in this study. The first version requires that the argument of the exponential martingale be evaluated through a stochastic Taylor expansion, which in turn necessitates the evaluation of multiple stochastic integrals along with the attendant requirement that the nonlinear terms be sufficiently differentiable. The second version, on the other hand, employs a new Monte Carlo-based strategy and evaluates the exponential correction to (virtually) any desired order or accuracy. Moreover, it requires no Taylor expansion or differentiability assumptions on the nonlinear terms. The GLM is numerically illustrated for a few SDOF and MDOF nonlinear oscillators with polynomial nonlinearity. Comparisons of the GLMbased solutions with exact stationary solutions, whenever available, are also provided.

Since the GLM propagates the solution purely through exponential transformations, it is readily exploitable as a Lie group method to help preserve any dynamical invariants that may be of interest. The GLM is also modifiable to achieve importance sampling and variance reduction with high precision. These issues constitute interesting elements of a future study.

\section{Acknowledgment}

The authors thankfully acknowledge the financial support from the Board of Research in Nuclear Sciences (BRNS), Government of India, through Grant No. DAEO/MCV/DR/112.

\section{Appendix}

Note that the first integral $\int_{0}^{t}\left(-\sigma^{-1}\left[K_{2} \widetilde{x}_{1}^{3}-K_{2} \tilde{x}_{1, i}^{3}\right]\right) d \hat{W}_{1}(s)$ on the right-hand side of Eq. (23) is an Ito integral and we will expand it using an Ito-Taylor expansion in terms of MSIs. By application of linearity property of Ito integral, we get

$$
\begin{aligned}
\int_{0}^{t}\left(-\sigma^{-1}\left[K_{2} \widetilde{x}_{1}^{3}-K_{2} \widetilde{x}_{1, i}^{3}\right]\right) d \hat{W}_{1}(s)= & -\sigma_{1}^{-1} K_{2} \int_{0}^{t}\left(\widetilde{x}_{1}^{3}\right) d \hat{W}_{1}(s) \\
& +\int_{0}^{t}\left(\sigma^{-1} K_{2} \widetilde{x}_{1, i}^{3}\right) d \hat{W}_{1}(s)
\end{aligned}
$$

Now we write the first term on the right-hand side of Eq. (A1) as

$$
-\sigma_{1}^{-1} K_{2} \int_{0}^{t}\left(\widetilde{x}_{1}^{3}\right) d \hat{W}_{1}(s)=-\sigma_{1}^{-1} K_{2} \times S I_{1}
$$

Applying Ito-Taylor expansion to $S I_{1}$ repeatedly, we get 


$$
\begin{aligned}
\Rightarrow S I_{1}= & \int_{0}^{t} \tilde{x}_{10}^{3} d \hat{W}_{1}(s)+3 \int_{0}^{t} \int_{0}^{s} \tilde{x}_{10}^{2} \tilde{x}_{20} d s_{1} d \hat{W}_{1}(s)+3 \int_{0}^{t} \int_{0}^{s}\left(-K_{2}\right) \tilde{x}_{10, i}^{3} d s_{1} d \hat{W}_{1}(s)+6 \int_{0}^{t} \int_{0}^{s} \int_{0}^{s_{1}}\left[\tilde{x}_{10} \tilde{x}_{20}^{2}+\int_{0}^{s_{2}}\left(\tilde{x}_{20}^{3}+2 \tilde{x}_{10} \tilde{x}_{20}\left\{-K_{1} \tilde{x}_{10}\right.\right.\right. \\
& \left.\left.-C_{1} \tilde{x}_{20}-K_{2} \tilde{x}_{10, i}^{3}\right\}+\tilde{x}_{10} \sigma_{1}^{2}\right) d s_{3}+2 \sigma_{1} \int_{0}^{s_{2}}\left(\tilde{x}_{10} \tilde{x}_{20}+\int_{0}^{s_{3}}\left(\tilde{x}_{20}^{3}+\tilde{x}_{10}\left\{-K_{1} \tilde{x}_{10}-C_{1} \tilde{x}_{20}-K_{2} \tilde{x}_{10, i}^{3}\right\}\right) d s_{4}\right. \\
& \left.\left.+\tilde{x}_{10} \int_{0}^{s_{3}} \sigma_{1} d \hat{W}_{1}\left(s_{4}\right)\right) d \hat{W}_{1}\left(s_{3}\right)\right] d s_{2} d s_{1} d \hat{W}_{1}(s)-3 K_{1} \int_{0}^{t} \int_{0}^{s} \int_{0}^{s_{1}}\left[\tilde{x}_{10}^{3}+3 \int_{0}^{s_{2}} \tilde{x}_{10}^{2} \tilde{x}_{20} d s_{3}\right] d s_{2} d s_{1} d \hat{W}_{1}(s) \\
& -3 C_{1} \int_{0}^{t} \int_{0}^{s} \int_{0}^{s_{1}}\left[\tilde{x}_{10}^{2} \tilde{x}_{20}+\int_{0}^{s_{2}}\left[2 \tilde{x}_{10} \tilde{x}_{20}^{2}+\tilde{x}_{10}^{2}\left\{-K_{1} \tilde{x}_{10}-C_{1} \tilde{x}_{20}-K_{2} \tilde{x}_{10, i}^{3}\right\}\right] d s_{3}+\sigma_{1} \int_{0}^{s_{2}}\left(\tilde{x}_{10}^{2}\right.\right. \\
& \left.\left.+2 \int_{0}^{s_{3}} \tilde{x}_{10} \tilde{x}_{20} d s_{4}\right) d \hat{W}_{1}\left(s_{3}\right)\right] d s_{2} d s_{1} d \hat{W}_{1}(s)+3 \sigma_{1} \int_{0}^{s} \int_{0}^{s} \int_{0}^{s_{1}}\left[\tilde{x}_{10}^{2}+2 \int_{0}^{s_{2}}\left(\tilde{x}_{10} \tilde{x}_{20}+\int_{0}^{s_{3}}\left(\tilde{x}_{20}^{3}+\tilde{x}_{10}\left\{-K_{1} \tilde{x}_{10}-C_{1} \tilde{x}_{20}-K_{2} \tilde{x}_{10, i}^{3}\right\}\right) d s_{4}\right.\right. \\
& \left.\left.+\tilde{x}_{10} \int_{0}^{s_{3}} \sigma_{1} d \hat{W}_{1}\left(s_{4}\right)\right) d s_{3}\right] d \hat{W}_{1}\left(s_{2}\right) d s_{1} d \hat{W}_{1}(s)+\cdots
\end{aligned}
$$

Now, the other integral on the right-hand side of Eq. (23) may also be expanded in a similar way as

$$
\begin{aligned}
\frac{1}{2} \int_{0}^{t}\left(-\sigma^{-1}\left[K_{2} \widetilde{x}_{1}^{3}-K_{2} \widetilde{x}_{1, i}^{3}\right]\right)^{2} d s= & \frac{1}{2} \sigma_{1}^{-2} K_{2}^{2} \int_{0}^{t} \widetilde{x}_{1}^{6} d s+\frac{1}{2} \sigma_{1}^{-2} K_{2}^{2} \widetilde{x}_{1, i}^{6} \int_{0}^{t} d s-\sigma_{1}^{-2} K_{2}^{2} \widetilde{x}_{1, i}^{3} \int_{0}^{t} \widetilde{x}_{1}^{3} d s=\frac{1}{2} \sigma_{1}^{-2} K_{2}^{2} \times S I_{2}+\frac{1}{2} \sigma_{1}^{-2} K_{2}^{2} \widetilde{x}_{1, i}^{6} t-\sigma_{1}^{-2} K_{2}^{2} \widetilde{x}_{1, i}^{3} \\
& \times S I_{3}
\end{aligned}
$$

Applying Ito-Taylor expansions to $\mathrm{SI}_{2}$ and $\mathrm{SI}_{3}$ repeatedly, we get

$$
\begin{aligned}
& S I_{2}=\int_{0}^{t} \widetilde{x}_{10}^{6} d s+6 \int_{0}^{t} \int_{0}^{s} \widetilde{x}_{10}^{5} \widetilde{x}_{20} d s_{1} d s+6 \int_{0}^{t} \int_{0}^{s}\left(-K_{2}\right) \widetilde{x}_{10, i}^{3} d s_{1} d s+30 \int_{0}^{t} \int_{0}^{s} \int_{0}^{s_{1}}\left[\widetilde{x}_{10}^{4} \widetilde{x}_{20}^{2}+\int_{0}^{s_{2}}\left(4 x_{10}^{3} \widetilde{x}_{20}^{3}+2 \widetilde{x}_{10}^{4} \tilde{x}_{20}\left\{-K_{1} \tilde{x}_{10}-C_{1} \widetilde{x}_{20}\right.\right.\right. \\
& \left.\left.\left.-K_{2} \widetilde{x}_{10, i}^{3}\right\}+\widetilde{x}_{10}^{4} \sigma_{1}^{2}\right) d s_{3}+\int_{0}^{s_{2}} 2 \widetilde{x}_{10}^{4} \tilde{x}_{20} \sigma_{1} d \hat{W}_{1}\left(s_{3}\right)\right] d s_{2} d s_{1} d s-6 K_{1} \int_{0}^{t} \int_{0}^{s} \int_{0}^{s_{1}}\left[\widetilde{x}_{10}^{6}+6 \int_{0}^{s_{2}} \tilde{x}_{10}^{5} \widetilde{x}_{20} d s_{3}\right] d s_{2} d s_{1} d s \\
& -6 C_{1} \int_{0}^{t} \int_{0}^{s} \int_{0}^{s_{1}}\left[\tilde{x}_{10}^{5} \tilde{x}_{20}+\int_{0}^{s_{2}}\left[5 \tilde{x}_{10}^{4} \tilde{x}_{20}^{2}+\widetilde{x}_{10}^{4}\left\{-K_{1} \tilde{x}_{10}-C_{1} \tilde{x}_{20}-K_{2} \tilde{x}_{10, i}^{3}\right\}\right] d s_{3}+\int_{0}^{s_{2}} \tilde{x}_{10}^{5} \sigma_{1} d \hat{W}_{1}\left(s_{3}\right)\right] d s_{2} d s_{1} d s+3 \sigma_{1} \int_{0}^{t} \int_{0}^{s} \int_{0}^{s_{1}}\left[\tilde{x}_{10}^{2}\right. \\
& \left.+\int_{0}^{s_{2}} 2 \tilde{x}_{10} \tilde{x}_{20} d s_{3}\right] d \hat{W}_{1}\left(s_{2}\right) d s_{1} d s+\ldots \\
& S I_{3}=\int_{0}^{t} \tilde{x}_{10}^{3} d s+3 \int_{0}^{t} \int_{0}^{s} \tilde{x}_{10}^{2} \tilde{x}_{20} d s_{1} d s+3 \int_{0}^{t} \int_{0}^{s}\left(-K_{2}\right) \widetilde{x}_{10, i}^{3} d s_{1} d s+6 \int_{0}^{t} \int_{0}^{s} \int_{0}^{s_{1}}\left[\tilde{x}_{10} \widetilde{x}_{20}^{2}+\int_{0}^{s_{2}}\left(\widetilde{x}_{20}^{3}+2 \tilde{x}_{10} \tilde{x}_{20}\left\{-K_{1} \tilde{x}_{10}-C_{1} \tilde{x}_{20}-K_{2} \widetilde{x}_{10, i}^{3}\right\}\right.\right. \\
& \left.\left.+\tilde{x}_{10} \sigma_{1}^{2}\right) d s_{3}+\int_{0}^{t} 2 \widetilde{x}_{10} \tilde{x}_{20} \sigma_{1} d \hat{W}_{1}\left(s_{3}\right)\right] d s_{2} d s_{1} d s-3 K_{1} \int_{0}^{t} \int_{0}^{s} \int_{0}^{s_{1}}\left[\tilde{x}_{10}^{3}+3 \int_{0}^{s_{2}} \widetilde{x}_{10}^{2} \tilde{x}_{20} d s_{3}\right] d s_{2} d s_{1} d s-3 C_{1} \int_{0}^{t} \int_{0}^{s} \int_{0}^{s_{1}}\left[\widetilde{x}_{10}^{2} \tilde{x}_{20}\right. \\
& \left.+\int_{0}^{s_{2}}\left[2 \widetilde{x}_{10} \widetilde{x}_{20}^{2}+\widetilde{x}_{10}^{2}\left\{-K_{1} \widetilde{x}_{10}-C_{1} \widetilde{x}_{20}-K_{2} \widetilde{x}_{10, i}^{3}\right\}\right] d s_{3}+\int_{0}^{t} \widetilde{x}_{10}^{2} \sigma_{1} d \hat{W}_{1}\left(s_{3}\right)\right] d s_{2} d s_{1} d s+3 \sigma_{1} \int_{0}^{t} \int_{0}^{s} \int_{0}^{s_{1}}\left[\tilde{x}_{10}^{2}\right. \\
& \left.+\int_{0}^{s_{2}} 2 \tilde{x}_{10} \tilde{x}_{20} d s_{3}\right] d \hat{W}_{1}\left(s_{2}\right) d s_{1} d s+\cdots
\end{aligned}
$$

The MSIs present in $\mathrm{SI}_{3}$ are the same as those in $\mathrm{SI}_{2}$. The MSIs are zero-mean Gaussian variables. See Roy and Dash [9] for details of evaluating the covariance matrix for MSIs.

\section{References}

[1] Lin, Y. K., and Cai, G. Q., 1988, "Exact Stationary Response Solution for Second Order Nonlinear Systems Under Parametric and External White Noise Excitations: Part II,” ASME J. Appl. Mech., 55, pp. 702-705.

[2] Kloeden, P. E., and Platen, E., 1999, Numerical Solution of Stochastic Differential Equations, Springer, New York.
[3] Milstein, G. N., 1995, Numerical Integration of Stochastic Differential Equations, Kluwer, Dordrecht.

[4] Maruyama, G., 1955, "Continuous Markov Processes and Stochastic Equations," Rend. Circ. Mat. Palermo, 4, pp. 48-90.

[5] Gard, T. C., 1988, Introduction to Stochastic Differential Equations, Marcel Dekker, New York.

[6] Rumelin, W., 1982, "Numerical Treatment of Stochastic Differential Equations," SIAM (Soc. Ind. Appl. Math.) J. Numer. Anal., 19(3), pp. 604-613.

[7] Burrage, K., Burrage, P., and Tian, T., 2004, "Numerical Methods for Strong Solutions of Stochastic Differential Equations: An Overview," Proc. R. Soc. London, Ser. A, 460(2041), pp. 373-402.

[8] Tocino, A., and Vigo-Aguiar, J., 2002, "Weak Second Order Conditions for 
Stochastic Runge-Kutta Methods,” SIAM J. Sci. Comput. (USA), 24(2), pp. 507-523.

[9] Roy, D., and Dash, M. K., 2005, "Explorations of a Family of Stochastic Newmark Methods in Engineering Dynamics," Comput. Methods Appl. Mech. Eng., 194(45-47), pp. 4758-4796.

[10] Roy, D., 2006, "A Family of Weak Stochastic Newmark Methods for Simplified and Efficient Monte Carlo Simulations of Oscillators," Int. J. Numer. Methods Eng., 67(3), pp. 364-399.

[11] Burrage, K., and Tian, T., 2004, "Implicit Stochastic Runge-Kutta Methods for Stochastic Differential Equations," BIT Numer. Math., 44(1), pp. 21-39.

[12] Milstein, G. N., Platen, E., and Schurz, H., 1998, "Balanced Implicit Methods for Stiff Stochastic Systems," SIAM (Soc. Ind. Appl. Math.) J. Numer. Anal., 35(3), pp. 1010-1019.

[13] Oksendal, B., 2004, Stochastic Differential Equations-An Introduction With Applications, 6th ed., Springer, New York.

[14] Socha, L., 2005, "Linearization in Analysis of Nonlinear Stochastic Systems: Recent Results-Part I: Theory,” Appl. Mech. Rev., 58(3), pp. 178-205.

[15] Socha, L., 2005, "Linearization in Analysis of Nonlinear Stochastic Systems: Recent Results-Part II: Applications," Appl. Mech. Rev., 58(5), pp. 303353.

[16] Socha, L., and Pawleta, M., 1994, "Corrected Equivalent Linearization," Mach. Dyn. Probl., 7, pp. 149-161.

[17] Elishakoff, I., and Colojani, P., 1997, "Stochastic Linearization Critically Reexamined," Chaos, Solitons Fractals, 8(12), pp. 1957-1972.

[18] Crandall, S. H., 2001, "Is Stochastic Equivalent Linearization a Subtly Flawed Procedure," Probab. Eng. Mech., 16(2), pp. 169-176.

[19] Falsone, G., and Elishakoff, I., 1994, "Modified Stochastic Linearization Technique for Coloured Noise Excitation of Duffing Oscillator," Int. J. Non-Linear Mech., 29(1), pp. 65-69.

[20] Apetaur, M., and Opicka, F., 1983, "Linearization of Nonlinear Stochastically Excited Dynamic Systems," J. Sound Vib., 86(4), pp. (563-585).

[21] Socha, L., 1999, "Statistical and Equivalent Linearization Techniques With Probability Density Criteria," J. Theor. Appl. Mech., 37, pp. 369-382.

[22] Anh, N. D., and Hung, L. X., 2003, "An Improved Criterion of Gaussian Equivalent Linearization for Analysis of Nonlinear Stochastic Systems," J. Sound Vib., 268(1), pp. 177-200.
[23] Kazakov, I. E., 1998, "An Extension of the Method of Statistical Linearization," Avtom. Telemekh., 59, pp. 220-224.

[24] Grundmann, H., Hartmann, C., and Waubke, H., 1998, "Structures Subjected to Stationary Stochastic Loadings. Preliminary Assessment by Statistical Linearization Combined With an Evolutionary Algorithm," Comput. Struct., 67(1-3), pp. 53-64.

[25] Iyengar, R. N., and Roy, D., 1996, "Conditional Linearization in Nonlinear Random Vibration," J. Eng. Mech., 122(3), pp. 197-200

[26] Roy, D., 2000, "Exploration of the Phase-Space Linearization Method for Deterministic and Stochastic Nonlinear Dynamical Systems," Nonlinear Dyn., 23(3), pp. 225-258

[27] Roy, D., 2001, "A New Numeric-Analytical Principle for Nonlinear Deterministic and Stochastic Dynamical Systems," Proc. R. Soc. London, Ser. A 457(2007), pp. 539-566.

[28] Roy, D., 2004, "A Family of Lower- and Higher-Order Transversal Linearization Techniques in Non-Linear Stochastic Engineering Dynamics," Int. J. Numer. Methods Eng., 61(5), pp. 764-790.

[29] Ibrahim, R. A., 1978, "Stationary Response of a Randomly Parametric Excited Nonlinear System,” ASME J. Appl. Mech., 45, pp. 910-916.

[30] Zhu, W. Q., 1988, "Stochastic Averaging Methods in Random Vibration" Appl. Mech. Rev., 41, pp. 189-199.

[31] Bernard, P., and Wu, L., 1998, "Stochastic Linearization: The Theory," J Appl. Probab., 35(3), pp. 718-730.

[32] Rubinstein, R. Y., 1981, Simulation and the Monte Carlo Method, Wiley, New York.

[33] Yazici, A., Atlas, I., and Ergenc, T., 2005, "2d Polynomial Interpolation: A Symbolic Approach with Mathematica," Lect. Notes Comput. Sci., 3482, pp. $463-471$.

[34] Wang, R., and Zhang, Z., 2000, "Exact Stationary Solutions of the FokkerPlanck Equation for Nonlinear Oscillators Under Stochastic Parametric and External Excitations," Nonlinearity, 13(3), pp. 907-920.

[35] Roy, D., and Dash, M. K., 2002, "A Stochastic Newmark Method for Engineering Dynamical Systems" J. Sound Vib., 249(1), pp. 83-100.

[36] Roy, D., 2003, "A Weak Form of Stochastic Newmark Method With Applications to Engineering Dynamical Systems," Appl. Math. Model., 27(6), pp. 421-436. 